\title{
Third Order Lagrangians, Weyl Invariants \& Classical Trace Anomaly in Six Dimensions
}

\author{
Mehrdad Farhoudi* \\ Department of Physics, Shahid Beheshti University G.C., Evin, Tehran 19839, Iran
}

April 30, 2013

\begin{abstract}
We have proceeded the analogy (represented in our previous works) of the Einstein tensor and the alternative form of the Einstein field equations for the generic coefficients of the eight terms in the third order of the Lovelock Lagrangian. We have found the constraint between the coefficients into two forms, an independent and a dimensional dependent versions. Each form has three degrees of freedom, and not only the exact coefficients of the third order Lovelock Lagrangian do satisfy the two forms of the constraints, but also the two independent cubic of the Weyl tensor satisfy the independent constraint in six dimensions and yield the dimensional dependent version identically independent of the dimension. Then, we have introduced the most general effective expression for a total third order type Lagrangian with the homogeneity degree number three which includes the previous eight terms plus the new three ones among the all seventeen independent terms. We have proceeded the analogy for this combination, and have achieved the relevant constraint. We have shown that the expressions given in the literature as the third Weyl-invariant combination in six dimensions do satisfy this constraint. Thus, we suggest that these constraint relations to be considered as the necessary consistency conditions on the numerical coefficients that a Weylinvariant in six dimensions should satisfy. Finally, we have calculated the "classical" trace anomaly (an approach that was presented in our previous works) for the introduced total third order type Lagrangian and have achieved a general expression with four degrees of freedom in more than six dimensions (three degrees in six dimensions). Then, we have demonstrated that the resulted expression contains exactly the relevant coefficient of the Schwinger-DeWitt proper time method (that linked with the relevant heat kernel coefficient) in six dimensions, as a particular case. Of course, this result is a necessary consistency check, nevertheless our approach can be regarded as an alternative (perhaps simpler, and classical) derivation of the trace anomaly which also gives a general expression with the relevant degrees of freedom.
\end{abstract}

PACS number: 04.20. $-q ; 04.50 .+h ; 04.20 . C v ; 04.90 .+e$

Keywords: Higher Order Gravities; Non-Linear Lagrangians; Weyl Invariants; Heat Kernel Coefficients; Lovelock Lagrangian.

\section{Introduction}

In our previous work [1, we have highlighted that the splitting feature of the Einstein tensor (as the first term of the Lovelock tensor [2]) into two parts - namely the Ricci tensor and the term proportional to the curvature scalar - with the trace relation between them is a common feature of each homogeneous term in the Lovelock tensor. We have emphasized that, indeed, this property can been resulted through the variation procedure. Then, motivated by the principle of general covariance, we have shown that this property can be generalized, via a generalized trace operator which we have

\footnotetext{
*E-mail: m-farhoudi@sbu.ac.ir
} 
defined and denoted by the Trace notation (instead of the trace one), for any inhomogeneous EulerLagrange expression that can be spanned linearly in terms of homogeneous tensors. And, as an immediate application, we have demonstrated that the (whole) Lovelock tensor (which is constructed of terms with a mixture of different orders) analogizes the mathematical feature of the Einstein tensor, and hence, we have classified the Lovelock gravity as a generalized Einstein gravity.

Still motivated by the principle of general covariance, we have proceeded 3 . further the analogy and enforced the mathematical appearance of the alternative form of the Einstein field equations (as a covariant form for gravitational field equations) for the relevant alternative form of the Lovelock field equations. From this, we have found that the price for this analogy is to accept the existence of the trace anomaly of the energy-momentum tensor even in the classical treatments. Thus, we have actually denoted [3] a classical view of gravitation which explicitly shows the presence of an extra anomalous trace for the energy-momentum tensor with an indication of the constitution of the higher order gravities towards it, exactly as what has been verified [4, 5, 6] in the quantum aspects of gravity. Indeed, in the quantum theory, one relates and justifies the presence of the trace anomaly, classically, to higher order gravities [7]. That is, the higher-order terms in curvature invariants have to be added to the effective Lagrangian of gravitational field when quantum corrections are considered, see, e.g., Ref. [8] and the beginning of Sect. 2. It has also been realized that such corrective terms are inescapable in order to obtain the effective quantum gravity action near the Plank scale 9]. Nevertheless, it is possible to show that, via conformal transformations, the higher-order (and even non-minimally coupled) terms always correspond to the Einstein gravity plus one (or more than one) minimally coupled scalar field, see, e.g., Refs. 10, 11 and references therein. Also, We have interpreted [10] that our classical procedure indicates compatibility with the Mach idea.

Then, we have employed [3] this procedure for any generic coefficients of the second order term of the Lovelock Lagrangian. And thus, we have gotten the resulted trace anomaly relation exactly the same as the trace anomaly consistency constraint which had been suggested by Duff [12] in any dimension. In addition to the second term of the Lovelock Lagrangian, the action constructed by the square of the Weyl conformal tensor, which is the only local geometrical conformal invariant in four dimensions, also satisfies this constraint 11 relation (only) in four dimensions (note that, the Weyl tensor is itself identically zero in three dimensions). This exact equality, between the trace anomaly relation suggested by Duff and the constraint relation that the constant coefficients of any generic second order Lagrangian must satisfy in order to hold the desired analogy, indicates that there may exist an intriguing interplay here. Indeed, one can speculate that an intrinsic reason for the existence of such a relation should perhaps be, classically, somehow related to the covariance 2 of the form of Einstein's equations [3. That is, by employing the generalized trace relation, the appearance of the trace anomaly may be interpreted as the Lovelock modification of gravity even in the classical treatments. Though it is somehow a naive conjecture, nevertheless, it gives almost an easy classical procedure to grasp the desired result which, besides what have been already given in the literature 14, 16, 17, 18, indicates an intrinsic property behind it. Hence, as a main advantage of this procedure, we have suggested [10] that this analogous of the Einstein tensor can even be employed as a criteria in order to distinguish correct/legitimate metric theories of gravity which are either homogeneous functions, or linear combinations of different homogeneous functions, of the metric and its derivatives.

The analogy is also capable to provide dimensional dependent relations. Actually, we have derived a dimensional dependent version of the Duff trace anomaly relation in Ref. [10], in where we have also summarized the important achievements of the procedure. The Gauss-Bonnet and the Weyl squared

\footnotetext{
${ }^{1}$ According to the classification of Refs. [5, 13, based on the dimensional regularization and power counting, this constraint indicates that in four dimensions (and indeed, in even dimensions), the anomaly can have two contributions, a type A anomaly (the Euler density invariants) and a type B anomaly (built from the conformal invariants). Also, a purely algebraic classification (independent of any regularization scheme) of the structure of the Weyl anomalies in arbitrary space-time dimensions has been presented in Ref. [14].

${ }^{2}$ In the semi-classical approach of quantum gravity theory, which has been employed to deduce the trace anomalies, the conformal invariance is sacrificed [15] to the needs of the general covariance (though, in contrary, see Ref. [16]).
} 
terms do satisfy this dimensional dependent constraint relation in any dimension. This dimensional dependent version of the Duff trace anomaly relation has also been re-derived in Ref. [19] (their relation (7)) by classifying higher derivative theories of gravity whose the traced field equations have a reduced order.

Now, in this work, we propose to probe further the analogy for the field tensor of the corresponding generic coefficients of the third order term of the Lovelock Lagrangian, and then for any type of third order Lagrangian. For this purpose, we give a brief review on the trace idea, particularly on the definition of the generalized trace tool in the following section. In Sect. 3, we provide the necessary relations for the generic third order Lagrangian terms and their Euler-Lagrange expressions. Also, in addition to the eight linearly independent terms, which appear in the third order of the Lovelock Lagrangian, we specify the other linearly independent types of third order Lagrangian terms. Then, in Sect. 4, based on the desired analogy, we derive the constraint relations among the constituent coefficients of the generic third order Lagrangians in two forms, an independent and a dimensional dependent versions. Hence, we match these relations for the two linearly independent scalars formed by the cubic of the Weyl tensor. The other Weyl-invariants are investigated when we incorporate all types of third order Lagrangian terms in Sect. 5. Actually, we first probe the trace analogy for the most general effective expression that we will introduce as a total third order Lagrangian, and then examine the resulted constraint relations for the numerical coefficients of the Weyl-invariants. We indicate the classical view of the trace anomaly in six dimensions in Sect. 6 , in where we also demonstrate that it has an interesting similarity with the appropriate heat kernel coefficients. Conclusions are presented in the last section, and some necessary formulations for the metric variation of a few Lagrangian terms plus a few useful relations and some derivations are furnished in Appendixes A and B.

\section{The Trace Idea Review}

We follow the sign conventions of Wald [20] and, analogous to the Einstein field equations, we assume that the geometry is proportional to the matter in a way that the gravitational field equations should be $G_{\alpha \beta}^{\text {(gravi.) }}=\kappa^{2} T_{\alpha \beta} / 2$, where the gravitational tensor, $G_{\alpha \beta}^{\text {(gravi.) }}$, represents the geometry, $\kappa^{2} \equiv 16 \pi G / c^{4}$ is the constant of the proportionality and the lower case Greek indices run from zero to $D-1$ in a $D$-dimensional space-time. Actually, the gravitational tensor is the Euler-Lagrange expression given in the process of the metric variation of the action, $\int L^{\text {(gravi.) }} \sqrt{-g} d^{D} x$, i.e.

$$
\delta\left[L^{\text {(gravi.) }} \sqrt{-g}\right]=\left[\frac{\delta L^{\text {(gravi.) }}}{\delta g^{\alpha \beta}}-\frac{1}{2} g_{\alpha \beta} L^{\text {(gravi.) }}\right] \sqrt{-g} \delta g^{\alpha \beta}=\frac{1}{\kappa^{2}} G_{\alpha \beta}^{\text {(gravi.) }} \sqrt{-g} \delta g^{\alpha \beta} .
$$

Also, analogous to the Einstein tensor, we demand

$$
G_{\alpha \beta}^{\text {(gravi.) }}=R_{\alpha \beta}^{\text {(gravi.) }}-\frac{1}{2} g_{\alpha \beta} R^{\text {(gravi.) }}
$$

with the trace relation between its two parts, exactly the same as the one yields between the Ricci tensor and the Ricci scalar.

In addition to the Einstein tensor, the Lovelock tensor has also been employed as a gravitational tensor. For example, the superstring theory, in its low energy limit, suggests that the EinsteinHilbert action should be enlarged by the inclusion of higher order curvature terms, and in order to be ghost-free it has been shown [21] that it must be in the form of dimensionally continued GaussBonnet densities (i.e., the Lovelock Lagrangian terms). An important aspect of this suggestion is 22 that it does not arise in attempts to quantize gravity. The ghost-free property and the fact that the Lovelock Lagrangian is the most general Lagrangian which (the same as the Einstein-Hilbert Lagrangian) yields the field equations as second order equations, have stimulated interests in the 
Lovelock gravity and its applications in the literature? The Lovelock Lagrangian is [2]

$$
L^{\text {(Lovelock) }}=\frac{1}{\kappa^{2}} \sum_{0<n<\frac{D}{2}} \frac{1}{2^{n}} c_{n} \delta_{\beta_{1} \ldots \beta_{2 n}}^{\alpha_{1} \ldots \alpha_{2 n}} R_{\alpha_{1} \alpha_{2}}^{\beta_{1} \beta_{2}} \cdots R_{\alpha_{2 n-1} \alpha_{2 n}}^{\beta_{2 n-1} \beta_{2 n}} \equiv \sum_{0<n<\frac{D}{2}} c_{n} L^{(n)},
$$

where we set $c_{1} \equiv 1$ and the other $c_{n}$ constants are of the order of the Planck length, $\ell_{P}=\sqrt{\hbar G / c^{3}}$, to the power $2(n-1)$, for making the dimension of $L^{\text {(Lovelock) }}$ to be the same as $L^{(1)} \equiv L_{\mathrm{E}-\mathrm{H}}=R / \kappa^{2}$. The $\delta_{\beta_{1} \ldots \beta_{p}}^{\alpha_{1} \ldots \alpha_{p}}$ is the generalized Kronecker delta symbol, which is identically zero if $p>D$, and in relation (3), the extremum value of $n$ is related to the dimension of space-time by

$$
n_{\text {ext. }}= \begin{cases}\frac{D}{2}-1 & \text { even } D \\ \frac{D-1}{2} & \text { odd } D .\end{cases}
$$

Hence, the $L^{\text {(Lovelock) }}$ reduces to the Einstein-Hilbert Lagrangian in four dimensions, and its second term is the Gauss-Bonnet invariant. The Lovelock tensor, as dimensionally reduction Euler-Lagrange terms, is [2]

$$
G_{\alpha \beta}^{(\text {Lovelock })}=-\sum_{0<n<\frac{D}{2}} \frac{1}{2^{n+1}} c_{n} g_{\alpha \mu} \delta_{\beta \beta_{1} \ldots \beta_{2 n}}^{\mu \alpha_{1} \ldots \alpha_{2 n}} R_{\alpha_{1} \alpha_{2}}^{\beta_{1} \beta_{2}} \cdots R_{\alpha_{2 n-1} \alpha_{2 n}}^{\beta_{2 n-1} \beta_{2 n}} \equiv \sum_{0<n<\frac{D}{2}} c_{n} G_{\alpha \beta}^{(n)},
$$

where the cosmological term has been neglected and the $G_{\alpha \beta}^{(1)} \equiv G_{\alpha \beta}$, i.e. the Einstein tensor. Relation

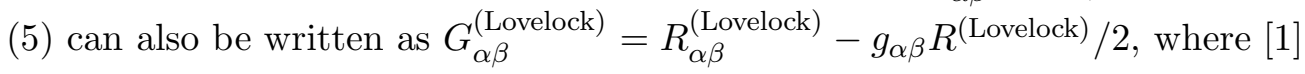

$$
R_{\alpha \beta}^{(\text {Lovelock })} \equiv \sum_{0<n<\frac{D}{2}} c_{n} R_{\alpha \beta}^{(n)} \quad \text { and } \quad R^{(\text {Lovelock })}=\kappa^{2} L^{(\text {Lovelock })} \equiv \sum_{0<n<\frac{D}{2}} c_{n} R^{(n)},
$$

with $R_{\alpha \beta}^{(n)}$ defined as

$$
R_{\alpha \beta}^{(n)} \equiv \frac{n}{2^{n}} \delta_{\alpha \beta_{2} \ldots \beta_{2 n}}^{\alpha_{1} \alpha_{2} \ldots \alpha_{2 n}} R_{\alpha_{1} \alpha_{2} \beta^{\beta_{2}}} R_{\alpha_{3} \alpha_{4}}^{\beta_{3} \beta_{4}} \cdots R_{\alpha_{2 n-1} \alpha_{2 n}{ }^{\beta_{2 n-1} \beta_{2 n}}}
$$

where also $R_{\alpha \beta}^{(1)} \equiv R_{\alpha \beta}$ and $R^{(1)} \equiv R$. With the usual definition of trace (i.e., the standard contraction of any two indices), one obtains [1] trace $R_{\alpha \beta}^{(n)} / n=R^{(n)}$, hence one cannot achieve a similar trace relation for the two parts of the whole Lovelock tensor. Nevertheless, we have shown [1] that with the generalized trace (denoted by the Trace, as distinct from the trace) operator, defined as follows, one can modify the original form of the trace relation adequately, and achieves $\operatorname{Trace} R_{\alpha \beta}^{(n)}=R^{(n)}$, hence $\operatorname{Trace} R_{\alpha \beta}^{\text {(Lovelock) }}=R^{\text {(Lovelock) }}$.

For a general $\left(\begin{array}{l}N \\ M\end{array}\right)$ tensor which is a homogeneous function of degree $h$ with respect to the metric and its derivatives (denoted in the brackets attached to the upper left-hand side of the tensor), we have defined [1]

$$
\operatorname{Trace}^{[h]} A^{\alpha_{1} \ldots \alpha_{N}}{ }_{\beta_{1} \ldots \beta_{M}}:= \begin{cases}\frac{1}{h-\frac{N}{2}+\frac{M}{2}} \operatorname{trace}^{[h]} A^{\alpha_{1} \ldots \alpha_{N}} \beta_{1} \ldots \beta_{M} & \text { when } h-\frac{N}{2}+\frac{M}{2} \neq 0 \\ \operatorname{trace}^{[h]} A^{\alpha_{1} \ldots \alpha_{N}} \beta_{1} \ldots \beta_{M} & \text { when } h-\frac{N}{2}+\frac{M}{2}=0,\end{cases}
$$

where, without loss of generality, the homogeneity degree number (HDN) conventions of each of $g^{\mu \nu}$ and $g^{\mu \nu}{ }_{, \alpha}$ are taken to be one. The HDN of a term consisted of cross functions is obviously found by adding the HDN of each of the cross functions. Hence, for example for two specified cross functions with the HDN $h^{\prime}$ and $h$, when $h^{\prime}+h \neq-1$ and $h^{\prime} \neq 0$, one gets

$$
\text { Trace }\left({ }^{\left[h^{\prime}\right]} C^{[h]} A_{\mu \nu}\right)= \begin{cases}\frac{h+1}{h^{h}+h+1}{ }^{\left[h^{\prime}\right]} C \operatorname{Trace}^{[h]} A_{\mu \nu} & \text { for } h \neq-1 \\ \frac{1}{h^{\prime}}\left[h^{\prime}\right] C \operatorname{Trace}^{[h]} A_{\mu \nu} & \text { for } h=-1\end{cases}
$$

\footnotetext{
${ }^{3}$ For review on the inclusion of higher order Lagrangians see, e.g., Refs. [10, 11, 23] and references therein.
} 
and when $h^{\prime}+h=-1$ and $h^{\prime} \neq 0$, one has

$$
\text { Trace }\left({ }^{\left[h^{\prime}\right]} C^{[h]} A_{\mu \nu}\right)=(h+1){ }^{\left[h^{\prime}\right]} C \text { Trace }{ }^{[h]} A_{\mu \nu} .
$$

Note that, as a homogeneous Euler-Lagrange expression has a uniform HDN, then one can work with the usual trace instead of the generalized trace operator. However, the notion of the generalized trace operator has been introduced to be effective when one considers the Einstein-Hilbert Lagrangian plus higher order terms as a complete gravitational Lagrangian, i.e. when one works with an inhomogeneous Lagrangian constructed linearly in terms of homogeneous terms.

\section{Third Order Lagrangian Terms}

The third order Lagrangian of the Lovelock Lagrangian is [24, 25, 26]

$$
L^{(3)}=\frac{1}{\kappa^{2}}\left(K_{1}-12 K_{2}+3 K_{3}+16 K_{4}+24 K_{5}-24 K_{6}+2 K_{7}-8 K_{8}\right),
$$

where 4

$$
\begin{array}{lll}
K_{1} \equiv R^{3}, & K_{2} \equiv R R_{\mu \nu} R^{\mu \nu}, & K_{3} \equiv R R_{\rho \tau \mu \nu} R^{\rho \tau \mu \nu}, \\
K_{4} \equiv R^{\mu \nu} R_{\mu \gamma} R_{\nu}{ }^{\gamma}, & K_{5} \equiv R_{\rho \tau} R_{\mu \nu} R^{\rho \mu \tau \nu}, & K_{6} \equiv R_{\lambda \rho} R^{\lambda \tau \mu \nu} R^{\rho}{ }_{\tau \mu \nu}, \\
K_{7} \equiv R^{\sigma \tau}{ }_{\mu \nu} R^{\mu \nu}{ }_{\lambda \rho} R^{\lambda \rho}{ }_{\sigma \tau}, & K_{8} \equiv R^{\sigma \tau}{ }_{\mu \nu} R^{\mu \lambda}{ }_{\sigma \rho} R^{\nu \rho}{ }_{\tau \lambda} . &
\end{array}
$$

These third order terms are the only linearly independent scalar terms, and the corresponding third order generic Lagrangian can be written as

$$
L_{\text {generic }}^{(3)}=\frac{1}{\kappa^{2}}\left(b_{1} K_{1}+b_{2} K_{2}+b_{3} K_{3}+b_{4} K_{4}+b_{5} K_{5}+b_{6} K_{6}+b_{7} K_{7}+b_{8} K_{8}\right),
$$

where the $b_{i}$ 's are arbitrary dimensionless constants, and obviously, in six dimensions, only seven of these eight terms are effective (see identity (37)). From definition (5), the third term of the Lovelock tensor is [24, 26]

$$
\begin{aligned}
G_{\alpha \beta}^{(3)}=3\{ & R^{2} R_{\alpha \beta}-4\left(R R_{\alpha \mu} R_{\beta}{ }^{\mu}+R R_{\alpha \mu \beta \nu} R^{\mu \nu}+R_{\alpha \beta} R_{\mu \nu} R^{\mu \nu}\right) \\
& +\left(2 R R_{\alpha \rho \mu \nu} R_{\beta}{ }^{\rho \mu \nu}+R_{\alpha \beta} R_{\rho \tau \mu \nu} R^{\rho \tau \mu \nu}\right)+8\left(R_{\alpha \mu} R_{\beta \nu} R^{\mu \nu}\right. \\
& \left.-R_{\alpha \mu \nu \beta} R^{\mu \rho} R^{\nu}{ }_{\rho}\right)-8\left[\left(R_{\alpha \mu} R_{\rho \tau} R_{\beta}{ }^{\rho \tau \mu}+\alpha \leftrightarrow \beta\right)-R_{\rho \alpha \beta \tau} R^{\rho \mu \nu \tau} R_{\mu \nu}\right] \\
& -4\left[\left(R_{\alpha \mu} R_{\beta \nu \rho \tau} R^{\mu \nu \rho \tau}+\alpha \leftrightarrow \beta\right)+R_{\alpha \mu \rho \tau} R_{\beta \nu}{ }^{\rho \tau} R^{\mu \nu}\right. \\
& \left.+2 R_{\alpha \rho \tau \mu} R_{\beta}{ }^{\rho \tau}{ }_{\nu} R^{\mu \nu}+R_{\alpha \mu \beta \nu} R^{\mu \rho \tau \lambda} R^{\nu}{ }_{\rho \tau \lambda}\right]+2 R_{\alpha \lambda \mu \nu} R_{\beta}{ }_{\rho \tau} R^{\mu \nu \rho \tau} \\
& \left.+8 R_{\alpha \mu \nu \lambda} R_{\beta \rho \tau}{ }^{\lambda} R^{\mu \tau \rho \nu}\right\}-\frac{1}{2} g_{\alpha \beta} \kappa^{2} L^{(3)}
\end{aligned}
$$

After some rather bulky calculations, the full appearance for the Euler-Lagrange expression of the third order generic Lagrangian can be written either as

$$
G_{(\text {generic }) \alpha \beta}^{(3 a)}=3\left(N_{\alpha \beta}+H_{\alpha \beta}\right)-\frac{1}{2} g_{\alpha \beta}\left(\kappa^{2} L_{\text {generic }}^{(3)}+M^{(3)}\right),
$$

or, inspired from the Euler-Lagrange expression (1) and assumptions

$$
\delta L^{\text {(gravi.) }} / \delta g^{\alpha \beta} \equiv R_{\alpha \beta}^{\text {(gravi.) }} / \kappa^{2} \quad \text { and } \quad L^{\text {(gravi.) }} \equiv R^{\text {(gravi.) }} / \kappa^{2},
$$

\footnotetext{
${ }^{4}$ Any other relevant term can easily be written in terms of these eight terms, e.g.$$
R_{\mu \nu}^{\sigma \tau} R_{\sigma \rho}^{\mu \lambda} R_{\lambda \tau}^{\nu}=K_{7} / 4+K_{8} .
$$

Actually, according to Ref. [27, the dimension of the basis of local cubic invariants with the Riemann tensor (without derivatives) is eight for $D>5$ dimensions.
} 
as

where $[26]$

$$
G_{\text {(generic) } \alpha \beta}^{(3 b)}=3\left(N_{\alpha \beta}+H_{\alpha \beta}-\frac{1}{6} g_{\alpha \beta} M^{(3)}\right)-\frac{1}{2} \kappa^{2} g_{\alpha \beta} L_{\text {generic }}^{(3)},
$$

$$
\begin{aligned}
M^{(3) \equiv} & \left(-3 b_{4}+2 b_{5}\right)\left(K_{4}-K_{5}\right)+\left(b_{5}+b_{6}\right)\left(K_{6}-K_{7}-2 K_{8}\right) \\
& -\left(12 b_{1}+b_{2}\right) K_{9}-\left(4 b_{2}+2 b_{5}\right) K_{10}-\left(4 b_{3}+\frac{1}{2} b_{5}+b_{6}\right) K_{11} \\
& -\left(2 b_{2}+3 b_{4}-b_{5}\right) K_{12}-\left(3 b_{4}-4 b_{5}-2 b_{6}\right) K_{13}-\left(12 b_{1}+2 b_{2}+\frac{3}{4} b_{4}\right) K_{15} \\
& -\left(4 b_{2}+4 b_{5}+2 b_{6}\right) K_{16}-\left(4 b_{3}+\frac{1}{2} b_{6}\right) K_{17} \\
N_{\alpha \beta} \equiv & b_{1} R^{2} R_{\alpha \beta}-\frac{4}{3} b_{3} R R_{\alpha \rho} R_{\beta}{ }^{\rho}+\frac{2}{3}\left(b_{2}+2 b_{3}\right) R R^{\mu \nu} R_{\alpha \mu \beta \nu}+\frac{1}{3} b_{2} R_{\alpha \beta} R^{\mu \nu} R_{\mu \nu} \\
& +\frac{2}{3} b_{3} R R_{\alpha \lambda \rho \sigma} R_{\beta}^{\lambda \rho \sigma}+\frac{1}{3} b_{3} R_{\alpha \beta} R^{\lambda \rho \sigma \tau} R_{\lambda \rho \sigma \tau}-\frac{1}{3}\left(b_{5}+2 b_{6}\right) R_{\alpha \mu} R_{\beta \nu} R^{\mu \nu} \\
& -\left(\frac{2}{3} b_{5}+\frac{2}{3} b_{6}-b_{8}\right) R_{\alpha \mu \nu \beta} R^{\mu \rho} R_{\rho}^{\nu}-\left(\frac{1}{2} b_{4}+\frac{1}{3} b_{5}+\frac{1}{3} b_{6}\right)\left(R_{\alpha \mu} R_{\lambda \rho} R_{\beta}{ }^{\lambda \mu}\right. \\
& +\alpha \leftrightarrow \beta)+\left(-\frac{2}{3} b_{6}+b_{8}\right) R_{\lambda \alpha \beta \rho} R^{\lambda \mu \nu \rho} R_{\mu \nu}-2 b_{7}\left(R_{\alpha \mu} R_{\beta \lambda \rho \sigma} R^{\mu \lambda \rho \sigma}\right. \\
& +\alpha \leftrightarrow \beta)+\left(\frac{2}{3} b_{6}+6 b_{7}\right) R_{\alpha \mu \lambda \rho} R_{\beta \nu}{ }^{\lambda \rho} R^{\mu \nu}+\left(\frac{2}{3} b_{6}-b_{8}\right) R_{\alpha \lambda \rho \mu} R_{\beta}{ }_{\nu} R_{\nu}^{\mu \nu} \\
& +\left(\frac{1}{3} b_{6}-\frac{1}{2} b_{8}\right) R_{\alpha \mu \beta \nu} R^{\mu \lambda \rho \sigma} R_{\lambda \rho \sigma}^{\nu}+\left(-\frac{2}{3} b_{6}-b_{7}+\frac{3}{2} b_{8}\right) R_{\alpha \tau \mu \nu} R_{\beta}{ }_{\lambda \rho} R^{\mu \nu \lambda \rho} \\
& +\left(\frac{4}{3} b_{6}+4 b_{7}-4 b_{8}\right) R_{\alpha \mu \nu \tau} R_{\beta \lambda \rho}{ }^{\tau} R^{\mu \rho \lambda \nu}
\end{aligned}
$$

and

$$
\begin{aligned}
H_{\alpha \beta} \equiv[ & -\left(b_{1}+\frac{1}{6} b_{2}+\frac{1}{24} b_{5}\right) R_{; \alpha} R_{; \beta}+\frac{1}{3}\left(b_{2}+4 b_{3}+\frac{1}{2} b_{5}+\frac{1}{2} b_{6}\right) R^{; \rho} R_{\alpha \beta ; \rho} \\
& -\left(b_{1}+\frac{1}{6} b_{2}+\frac{1}{3} b_{3}\right) R R_{; \alpha \beta}+\frac{1}{6}\left(b_{2}+4 b_{3}\right) R R_{\alpha \beta ; \rho}{ }^{\rho}+\frac{1}{6}\left(b_{2}+\frac{1}{2} b_{5}\right)\left(R_{; \rho}{ }^{\rho} R_{\alpha \beta}\right. \\
& \left.-2 R^{\mu \nu} R_{\mu \nu ; \alpha \beta}\right)-\left(\frac{2}{3} b_{3}+\frac{1}{6} b_{6}-\frac{1}{4} b_{8}\right)\left(R^{; \mu \nu} R_{\alpha \mu \nu \beta}+\frac{1}{2} R_{\lambda \rho \sigma \tau ; \alpha \beta} R^{\lambda \rho \sigma \tau}\right) \\
& -\left(\frac{1}{3} b_{2}+\frac{1}{3} b_{5}+\frac{1}{2} b_{8}\right) R_{\mu \nu ; \alpha} R^{\mu \nu} ; \beta+\left(\frac{1}{2} b_{4}+\frac{2}{3} b_{6}+2 b_{7}-\frac{1}{2} b_{8}\right) R_{\alpha \mu ; \nu} R_{\beta}^{\mu ; \nu} \\
& -\left(\frac{1}{6} b_{5}+\frac{1}{3} b_{6}+2 b_{7}\right) R_{\alpha \mu ; \nu} R_{\beta}^{\nu ; \mu}-\left(\frac{1}{3} b_{3}+\frac{1}{8} b_{8}\right) R_{\lambda \rho \sigma \tau ; \alpha} R^{\lambda \rho \sigma \tau} ; \beta \\
& +\frac{1}{3}\left(b_{5}+b_{6}\right)\left(R^{\mu \nu} R_{\alpha \beta ; \mu \nu}-R^{\mu \nu ; \rho} R_{\alpha \mu \rho \beta ; \nu}\right)-\left(\frac{1}{3} b_{5}+b_{8}\right)\left(\frac{1}{2} R_{\mu \nu ; \rho}{ }^{\rho} R_{\alpha}{ }^{\mu \nu} \beta\right. \\
& \left.-R_{\mu \nu ; \alpha \rho} R_{\beta}^{\mu \nu \rho}\right)+\left(\frac{1}{6} b_{6}+b_{7}-\frac{1}{4} b_{8}\right)\left(R_{\lambda \rho \sigma \alpha ; \tau} R^{\lambda \rho \tau} ; \sigma-4 R_{\alpha \rho ; \mu \nu} R_{\beta}{ }^{\mu \nu \rho}\right) \\
& +\left(\frac{1}{3} b_{6}-b_{8}\right) R^{\mu \nu ; \rho} R_{\alpha \mu \nu \rho ; \beta}-\left(\frac{1}{3} b_{2}+\frac{4}{3} b_{3}+\frac{1}{4} b_{4}+\frac{1}{6} b_{6}\right) R^{; \rho} R_{\alpha \rho ; \beta} \\
& -\left(\frac{1}{3} b_{2}+\frac{1}{4} b_{4}+\frac{1}{6} b_{5}+\frac{1}{6} b_{6}\right) R_{; \alpha}{ }^{\rho} R_{\beta \rho}-\left(\frac{1}{2} b_{4}-\frac{1}{3} b_{5}+\frac{1}{3} b_{6}-b_{8}\right) \times \\
& \left.R_{\alpha \mu ; \nu} R^{\mu \nu} ; \beta+\left(\frac{1}{2} b_{4}+\frac{1}{3} b_{6}\right)\left(R_{\alpha}^{\rho} R_{\beta \rho ; \mu}{ }^{\mu}-R^{\mu \nu} R_{\alpha \mu ; \beta \nu}\right)\right]+\alpha \leftrightarrow \beta
\end{aligned}
$$

As seen, in contrast to the $G_{\alpha \beta}^{(3)}$, the $G_{\text {(generic) } \alpha \beta}^{(3)}$ is up to the fourth order jet-prolongation of the metric, as expected6. The third and fourth order terms are due to the $H_{\alpha \beta}$ and $M^{(3)}$ terms, which will vanish if and only if the constant coefficients satisfy the ratios

$$
b_{2}=-12 b_{1}, \quad b_{3}=3 b_{1}, \quad b_{4}=16 b_{1}, \quad b_{5}=24 b_{1},
$$

\footnotetext{
${ }^{5}$ Also, see relation (A.19).

${ }^{6}$ For a few solutions (mainly the black hole solutions) to the curvature-cubed (sometimes also called the six derivative) interactions, see, e.g., Refs. 28.
} 


$$
b_{6}=-24 b_{1}, \quad b_{7}=2 b_{1}, \quad b_{8}=-8 b_{1} .
$$

These conditions are exactly the ratios of the constituent coefficients of the special case of $L^{(3)}$ that leads to the $G_{\alpha \beta}^{(3)}$. Also, with the above ratios, the $N_{\alpha \beta}$ term will be equal to the corresponding counterpart of the $G_{\alpha \beta}^{(3)}$.

To amend the Lagrangian of sixth order gravity 29, Berkin and et al. 30] discussed that the Lagrangian term of $R \square R$ is a third order Lagrangian based on the dimensionality scale 7 however, it can be better justified on account of its HDN which is three. Indeed, to classify different gravitational Lagrangian terms, it is straightforward to relate [1, 10] the order $n$ in any Lagrangian (as in the $L^{(n)}$ ) to represent its HDN and referring to Lagrangians with their HDNs rather than their orders. Hence, by gathering terms with the same HDN under one Lagrangian label, in addition to the eight linearly independent terms, in $D>5$ dimensions, which appear in the third order of the Lovelock Lagrangian (and are up to the second order jet-prolongation of the metric), there are [26, in general, another nine linearly independent scalar terms, constructed from the Riemann-Christoffel tensor and its contractions, with the HDN three which are up to the third or even higher order jet-prolongation of the metric. They all also satisfy the dimensionality scale, and are 8

$$
\begin{array}{lll}
K_{9} \equiv R \square R & K_{10} \equiv R_{\mu \nu} \square R^{\mu \nu} & K_{11} \equiv R_{\mu \nu \rho \tau} \square R^{\mu \nu \rho \tau} \\
K_{12} \equiv R_{\mu \nu} R^{; \mu \nu} & K_{13} \equiv R_{\mu \nu ; \rho} R^{\mu \rho ; \nu} & K_{14} \equiv \square^{2} R \\
K_{15} \equiv R_{; \rho} R^{; \rho} & K_{16} \equiv R_{\mu \nu ; \rho} R^{\mu \nu ; \rho} & K_{17} \equiv R_{\mu \nu \rho \tau ; \alpha} R^{\mu \nu \rho \tau ; \alpha},
\end{array}
$$

where $\square \equiv_{;} \rho^{\rho}$. Each of the $K_{i}$ 's gives a dimensionless action in six dimensions. Any other relevant term, e.g. $R_{\mu \nu ; \rho \tau} R^{\rho \mu \nu \tau}$, can be written in terms of these terms, see, e.g. the last relation of (A.1). Besides, not all of their corresponding Euler-Lagrange expressions are independent; however, see the Appendix A for the effects of these nine terms as scalar Lagrangians.

\section{$4 \quad L_{\text {generic }}^{(3)}$ With The Trace Property}

In this section, we investigate the analogy for the field tensor of the $L_{\text {generic }}^{(3)}$, and then, in the next section, for any type of third order Lagrangian including those mentioned in (23). The relevant Euler-Lagrange expression $G_{(\text {generic } \alpha \beta}^{(3)}$ has been written as relations (16) and (18); however, we also demand to have them as in (2) , i.e. $G_{(\text {generic }) \alpha \beta}^{(3)}=R_{(\text {generic }) \alpha \beta}^{(3)}-g_{\alpha \beta} R_{\text {generic }}^{(3)} / 2$, but propose to find out whether, and for what conditions, the relation

$$
\operatorname{Trace} R_{\text {(generic) } \alpha \beta}^{(3)}=R_{\text {generic }}^{(3)}
$$

can be valid. We carry out this investigation for both appearances of $G_{(\text {generic) } \alpha \beta}^{(3)}$ in the following two parts.

Part (a): The Case $G_{(\text {generic }) \alpha \beta}^{(3 a)}$

In this case, from (16), we have $R_{(\text {generic) }}^{(3 a)}=\kappa^{2} L_{\text {generic }}^{(3)}+M^{(3)}$ and

$$
\operatorname{Trace} R_{\text {(generic) } \alpha \beta}^{(3 a)}=3\left(\text { Trace } N_{\alpha \beta}+\text { Trace } H_{\alpha \beta}\right) .
$$

Using the definition of generalized trace and the fact that each of the $N_{\alpha \beta}$ and $H_{\alpha \beta}$, and hence the $R_{(\text {generic) } \alpha \beta}^{(3 a)}$, has the HDN two [1, we get

$$
\text { Trace } N_{\alpha \beta}=\frac{1}{3}\left[b_{1} K_{1}+b_{2} K_{2}+b_{3} K_{3}+\left(\frac{1}{3} b_{5}-b_{8}\right) K_{4}+\left(b_{4}+\frac{2}{3} b_{5}+b_{8}\right) K_{5}\right.
$$

\footnotetext{
${ }^{7}$ Since two derivatives are dimensionally equivalent to one Riemann-Christoffel tensor or any one of its contractions.

${ }^{8}$ See also Refs. 17, 27, 31.
} 


$$
\left.+\left(\frac{5}{3} b_{6}+2 b_{7}-\frac{3}{2} b_{8}\right) K_{6}+\left(-\frac{2}{3} b_{6}-b_{7}+\frac{3}{2} b_{8}\right) K_{7}+\left(-\frac{4}{3} b_{6}-4 b_{7}+4 b_{8}\right) K_{8}\right]
$$

and

$$
\begin{aligned}
\text { Trace } H_{\alpha \beta}=\frac{1}{3}[ & \left(\frac{1}{3} b_{5}-\frac{2}{3} b_{6}-4 b_{7}+2 b_{8}\right)\left(K_{6}-K_{7}-2 K_{8}\right)-\left(2 b_{1}-\frac{1}{3} b_{2}-\frac{2}{3} b_{3}\right. \\
& \left.-\frac{1}{6} b_{5}\right) K_{9}+\left(-\frac{2}{3} b_{2}+b_{4}+\frac{2}{3} b_{6}+b_{8}\right) K_{10}+\left(-\frac{2}{3} b_{3}-\frac{1}{6} b_{5}+\frac{1}{6} b_{6}\right. \\
& \left.+2 b_{7}-\frac{3}{4} b_{8}\right) K_{11}-\left(\frac{2}{3} b_{2}-\frac{4}{3} b_{3}+b_{4}-\frac{1}{3} b_{5}-\frac{1}{3} b_{6}+\frac{1}{2} b_{8}\right) K_{12} \\
& -\left(b_{4}-b_{5}+4 b_{7}\right) K_{13}+\left(-2 b_{1}+\frac{4}{3} b_{3}-\frac{1}{4} b_{4}+\frac{1}{4} b_{5}+\frac{1}{6} b_{6}\right) K_{15} \\
& \left.-\left(\frac{2}{3} b_{2}-b_{4}+\frac{2}{3} b_{5}-\frac{2}{3} b_{6}-4 b_{7}\right) K_{16}+\left(-\frac{2}{3} b_{3}+\frac{1}{6} b_{6}+b_{7}-\frac{1}{2} b_{8}\right) K_{17}\right] .
\end{aligned}
$$

Then, after all the necessary substitutions have been made, we find that the equality of (24) holds if and only if the following conditions between those eight non-zero constituent coefficients of $L_{\text {generic }}^{(3)}$ are satisfied. That is, the constant coefficients are not all independent but obey the constraint

$$
\begin{array}{ll}
b_{4}=2\left(-b_{2}+4 b_{3}\right) / 3, & b_{5}=-60 b_{1}-8 b_{2}-4 b_{3}, \\
b_{6}=30 b_{1}+2 b_{2}-10 b_{3}, & b_{7}=30 b_{1}+14 b_{2} / 3+28 b_{3} / 3, \\
b_{8}=100 b_{1}+12 b_{2}+12 b_{3} . &
\end{array}
$$

These conditions are satisfied for (as must be the case) the non-generic Lagrangian $L^{(3)}$, i.e. for the coefficients given by relation (22) 9 . Though, it is not only this Lagrangian that has the analogy of the Einstein tensor and, using constraint (28), there are more combinations for $L_{\text {generic }}^{(3)}$ with three degrees of freedom (out of its eight coefficients) that have the required trace property (see below for other examples).

As mentioned, the $L_{\text {generic }}^{(3)}$ is a Lagrangian which gives $G_{(\text {generic }) \alpha \beta}^{(3 a)}$ as in relation (16) with $R_{\text {generic }}^{(3 a)}=\kappa^{2} L_{\text {generic }}^{(3)}+M^{(3)} ;$ however, one can define 10

$$
\kappa^{2} L_{\text {generic }}^{\prime(3)} \equiv \kappa^{2} L_{\text {generic }}^{(3)}+M^{(3)},
$$

where, as is shown in the Appendix A, the term $M^{(3)}$ is a complete divergence (see relation (A.19) and will give no contribution to the variation of the relevant action. Therefore, this new Lagrangian, $L_{\text {generic }}^{\prime(3)}$, gives the same $G_{(\text {generic }) \alpha \beta}^{(3 a)}$ with $R_{\text {generic }}^{(3 a)} \equiv \kappa^{2} L_{\text {generic }}^{\prime(3)}$ which is analogous to the appearances of the case $L^{(3)}$, the complete Lovelock Lagrangian (relation (마) ) and the assumption (17).

If one wants to use different choices of $L_{\text {generic }}^{(3)}$, for which more than three of the constituent coefficients are zero (as, for example, not all of the terms in $L_{\text {generic }}^{(3)}$ have been given by the superstring theory 11 ), then the trace condition (28) will not be satisfied. Because, the maximum number of coefficients that it permits at a time to be equal to zero (otherwise all of them will be zero) are generally two, and only for the following special cases are three. Therefore, those choices of $L_{\text {generic }}^{(3)}$ that miss more than the permitted terms cannot provide the trace relation. The following two cases are the only cases in which three coefficients of the $L_{\text {generic }}^{(3)}$ can simultaneously be made zero, though there is still one degree of freedom remaining to satisfy the trace condition. If one chooses $b_{2}=0=b_{3}$, then constraint (28) will give

$$
b_{4}=0, \quad b_{5}=-60 b_{1}, \quad b_{6}=30 b_{1}, \quad b_{7}=30 b_{1}, \quad b_{8}=100 b_{1},
$$

\footnotetext{
${ }^{9}$ Just by choosing $b_{2}=-12 b_{1}$ and $b_{3}=3 b_{1}$ (as in (22)), constraint (28) then gives the remaining coefficients with the same ratios as those of (22).

${ }^{10}$ Note that, although this $L_{\text {generic }}^{\prime(3)}$ is again up to the fourth order (and not up to the second order) jet-prolongation of the metric, but its HDN is still three, the same as the $L_{\text {generic }}^{(3)}$ term, in agreement with our demand of gathering terms with the same HDN under one Lagrangian label.

${ }^{11}$ See, for example, Ref. 32].
} 
and if one sets $b_{2}=-20 b_{1} / 3$ and $b_{3}=-5 b_{1} / 3$, it then will read

$$
b_{4}=0, \quad b_{5}=0, \quad b_{6}=100 b_{1} / 3, \quad b_{7}=-50 b_{1} / 3, \quad b_{8}=0 .
$$

Similar to the Lagrangian constructed from the square of the Weyl tensor, relation (A.9), there are only twd 12 linearly independent scalars formed by the cubic of the Weyl tensor. We choose the $C^{\sigma \tau}{ }_{\mu \nu} C^{\mu \nu}{ }_{\lambda \rho} C^{\lambda \rho}{ }_{\sigma \tau}$ and $C^{\sigma \tau}{ }_{\mu \nu} C^{\mu \lambda}{ }_{\sigma \rho} C^{\nu \rho}{ }_{\tau \lambda}$ (with the indices similar to the ones of the $K_{7}$ and $K_{8}$ terms, respectively). Their expressions in $D \geq 3$ dimensions (also, see the end of the Useful Relations in the Appendix A) are

$$
\begin{aligned}
A_{1} \equiv C^{\sigma \tau}{ }_{\mu \nu} C^{\mu \nu}{ }_{\lambda \rho} C^{\lambda \rho}{ }_{\sigma \tau}= & \frac{8(2 D-3)}{(D-2)^{3}(D-1)^{2}} K_{1}-\frac{24(2 D-3)}{(D-2)^{3}(D-1)} K_{2}+\frac{6}{(D-2)(D-1)} K_{3} \\
& +\frac{16(D-1)}{(D-2)^{3}} K_{4}+\frac{24}{(D-2)^{2}} K_{5}-\frac{12}{(D-2)} K_{6}+K_{7}
\end{aligned}
$$

and

$$
\begin{aligned}
A_{2} \equiv C_{\mu \nu}^{\sigma \tau} C_{\sigma \rho}^{\mu \lambda} C_{\tau \lambda}^{\nu \rho}= & -\frac{D^{2}+5 D-10}{(D-2)^{3}(D-1)^{2}} K_{1}+\frac{3\left(D^{2}+5 D-10\right)}{(D-2)^{3}(D-1)} K_{2}-\frac{3}{(D-2)(D-1)} K_{3} \\
& -\frac{2(5 D-6)}{(D-2)^{3}} K_{4}-\frac{3(D+2)}{(D-2)^{2}} K_{5}+\frac{6}{(D-2)} K_{6}+K_{8} .
\end{aligned}
$$

The coefficients of the above expressions can satisfy constraint (28) only if $D=6$. Their values in this dimension respectively are 13

$$
\begin{array}{llll}
b_{1}=9 / 200, & b_{2}=-27 / 40, & b_{3}=3 / 10, & b_{4}=5 / 4, \\
b_{5}=3 / 2, & b_{6}=-3, & b_{7}=1, & b_{8}=0
\end{array}
$$

and

$$
\begin{array}{llll}
b_{1}=-7 / 200, & b_{2}=21 / 40, & b_{3}=-3 / 20, & b_{4}=-3 / 4, \\
b_{5}=-3 / 2, & b_{6}=3 / 2, & b_{7}=0, & b_{8}=1 .
\end{array}
$$

Alternatively, one can obviously write expression (33), in six dimensions, effectively (using identity (37)) with the values

$$
\begin{array}{llll}
b_{1}=9 / 100, & b_{2}=-39 / 40, & b_{3}=9 / 40, & b_{4}=5 / 4, \\
b_{5}=3 / 2, & b_{6}=-3 / 2, & b_{7}=1 / 4, & b_{8}=0 .
\end{array}
$$

The Lagrangian densities made by these two cubic constructions of the Weyl tensor are the only conformal (Weyl) invariant in six dimensions. However, according to Refs. [13, 36], in general, there are three linearly independent Weyl-invariant combinations in six dimensions, and two of them are obviously the above purely algebraic ones. The another one (given by 14 relation (47)) contains terms from the other nine third order Lagrangian ones mentioned in (23), and it is one of the reasons why we will extend the analogy further to consider any type of third order Lagrangian, including the terms in (23), in the next section.

In six dimensions, by the generalization of the Gauss-Bonnet theorem, the $L^{(3)} \sqrt{-g}$ corresponds to the Euler densitie 15 (i.e., it is a complete divergence), and hence one gets one more constraint among the coefficients $b_{1}$ to $b_{8}$ in this dimension. That is, in six dimensions, one has

$$
\left.\left(G_{\alpha \beta}^{\left(K_{1}\right)}-12 G_{\alpha \beta}^{\left(K_{2}\right)}+3 G_{\alpha \beta}^{\left(K_{3}\right)}+16 G_{\alpha \beta}^{\left(K_{4}\right)}+24 G_{\alpha \beta}^{\left(K_{5}\right)}-24 G_{\alpha \beta}^{\left(K_{6}\right)}+2 G_{\alpha \beta}^{\left(K_{7}\right)}-8 G_{\alpha \beta}^{\left(K_{8}\right)}\right)\right|_{6-\text { dim. }} \equiv 0 .
$$

\footnotetext{
${ }^{12}$ According to Ref. 33] and the appendix of Ref. 34, the dimension of the basis of local cubic Weyl-invariants is two for $D>5$ dimensions, as, e.g., in four dimensions, one has $A_{1}=4 A_{2}$ since $5 C^{\sigma \tau}{ }_{[\mu \nu} C^{\mu \nu}{ }_{\lambda \rho} C^{\lambda \rho}{ }_{\sigma] \tau}=A_{1}-4 A_{2}$.

${ }^{13}$ These values already are consistent with when the generalization of the Gauss-Bonnet theorem 35 in six dimensions has also been considered.

${ }^{14}$ However, see also relations (49), (50) and (54).

${ }^{15}$ See, for example, Ref. [17].
} 
Therefore, for the remaining seven linearly independent effective terms, after substituting for one of the term according to identity (37), constraint (28) for the new coefficients reduces to an effective one. For example, by substituting for the $K_{8}$ term, the effective constraint is

$$
\begin{array}{ll}
b_{1}=-3\left(b_{2}+b_{3}\right) / 25, & b_{4}=2\left(-b_{2}+4 b_{3}\right) / 3, \\
b_{5}=4\left(-b_{2}+4 b_{3}\right) / 5, & b_{6}=-4\left(2 b_{2}+17 b_{3}\right) / 5, \\
b_{7}=2\left(8 b_{2}+43 b_{3}\right) / 15, &
\end{array}
$$

with two degrees of freedom, where here we have chosen the $b_{2}$ and $b_{3}$ coefficients. Constraint (38) is satisfied by the values given in (34) and (36), as expected.

Part (b): The Case $G_{(\text {generic }) \alpha \beta}^{(3 b)}$

Now, we calculate the generalized trace of the first part of relation (18), knowing the fact that the HDN of the $M^{(3)}$ is three. By using relation (9), we get

$$
\operatorname{Trace} R_{(\text {generic }) \alpha \beta}^{(3 b)}=3\left(\operatorname{Trace} N_{\alpha \beta}+\text { Trace } H_{\alpha \beta}\right)-\frac{D}{6} M^{(3)},
$$

and again, after all the necessary substitutions and calculations have been performed, we find that the equality of (24), for non-zero coefficients, holds if and only if

$$
\begin{aligned}
& b_{4}=2\left(-b_{2}+4 b_{3}\right) / 3, \\
& b_{5}=-12(D-1) b_{1}-(D+2) b_{2}-4 b_{3}, \\
& b_{6}=6(D-1) b_{1}+2 b_{2}-2(D-1) b_{3}, \\
& b_{7}=3(D-2)(D-1) b_{1} / 2+(D-2)(D+1) b_{2} / 6+\left(D^{2}+5 D-10\right) b_{3} / 6, \\
& b_{8}=4(D-1)^{2} b_{1}+D^{2} b_{2} / 3+4(2 D-3) b_{3} / 3,
\end{aligned}
$$

where at least16 $D>3$. Though constraint (40) depends on the dimension of space-time, it still gives three degrees of freedom out of the eight coefficients. It is satisfied by the $L^{(3)}$ in any dimension. This can be obvious after arranging constraint (40) with respect to the dimension, $D$, and its powers for each coefficient. Then from these, one can easily find that the only combination which is independent of the dimension is that of the $L^{(3)}$.

Also, the coefficients of the scalar Lagrangians made by the cubic of the Weyl tensor, relations (32) and (33), identically satisfy constraint (40) independent of the dimension of space-time $D$.

As is evident, by comparing relations (25) and (39), constraint (40) in six dimensions is exactly the same as condition (28). The reason beyond it is that, suppose, in general, a $P$ scalar term is the necessary shifting term by which the two appearances, similar to part (a) and part (b) (i.e., similar to relations (16) and (18) $)$, are arranged. Hence, by comparing these two parts, e.g. for the relevant part similar to part (b), one obtains

$$
R_{\text {(generic) } \alpha \beta}^{(n b)} \equiv R_{(\text {generic }) \alpha \beta}^{(n a)}-g_{\alpha \beta} P \quad \text { and } \quad R_{\text {generic }}^{(n b)} \equiv R_{\text {generic }}^{(n a)}-2 P .
$$

Now, in order to achieve Trace $R_{(\text {generic }) \alpha \beta}^{(n a)}=R_{\text {generic }}^{(n a)}$ and $\operatorname{Trace} R_{(\text {generic }) \alpha \beta}^{(n b)}=R_{\text {generic }}^{(n b)}$ simultaneously be satisfied, we must have $\operatorname{Trace}\left(g_{\alpha \beta} P\right)=2 P$. If $P$ is a homogeneous function of degree $h$ with respect to the metri 17 then, using the generalized trace definition (8), one will get

$$
\begin{cases}D=2 h & \text { when } h \neq 0 \\ D=2 & \text { when } h=0 .\end{cases}
$$

In the case of $L_{\text {generic }}^{(2)}$, we have shown [10] that $P \propto \square R$, hence it has $h=2$ (as expected), and thus $D=4$. In the case of $L_{\text {generic }}^{(3)}, P$ is proportional to the $M^{(3)}$, therefore it has $h=3$ (again, as expected), and thus $D=6$.

\footnotetext{
${ }^{16}$ In three dimensions, there are other identities, e.g. relations A.10 and A.11), that should also be considered; see the appendix of Ref. 34] too.

${ }^{17}$ Though, according to the idea of gathering terms with the same HDN under one Lagrangian label, $P$ must be a homogeneous function of degree $n$ for the $L_{\text {generic }}^{(n)}$.
} 


\section{Other Weyl Invariants}

In this section, we extend the analogy to include the other nine third order Lagrangian terms in (23). In addition to the reason mentioned almost at the end of part (a), this extension is needed because, the conformal anomalies in six dimensions contain the term $K_{14}$ (see relation (75)) which appears only when the Lagrangian terms (23) are considered, see, e.g., relations (A.30) and (A.31). However, as explained in the Appendix A, it should suffice to consider the Lagrangian terms $K_{1}$ to $K_{10}$ (see relation (A.27) ) rather than the $K_{1}$ to $K_{17}$. Though, as will be illustrated below, in order to be able to apply the trace analogy to an effective total third order Lagrangian, one, in practice, needs to consider all of the $K_{9}, K_{10}$ and $K_{11}$ terms simultaneously in such a Lagrangian. Hence, by neglecting identity (A.20), we consider the most general effective expression for the total third order Lagrangian to $b e^{18}$

$$
L_{\text {total }}^{(3)} \stackrel{\text { eff }}{=} L_{\text {generic }}^{(3)}+\left(b_{9} K_{9}+b_{10} K_{10}+b_{11} K_{11}\right) / \kappa^{2} .
$$

Furthermore, we again want to write its corresponding Euler-Lagrange expression the same as (2), i.e. $G_{(\text {total }) \alpha \beta}^{(3 a)}=R_{(\text {total }) \alpha \beta}^{(3 a)}-g_{\alpha \beta} R_{\text {total }}^{(3 a)} / 2$, where 19

$$
G_{(\text {total }) \alpha \beta}^{(3 a)}=G_{(\text {generic }) \alpha \beta}^{(3 a)}+b_{9} G_{\alpha \beta}^{\left(K_{9}\right)}+b_{10} G_{\alpha \beta}^{\left(K_{10}\right)}+b_{11} G_{\alpha \beta}^{\left(K_{11}\right)}
$$

and similar expressions for $R_{(\text {total }) \alpha \beta}^{(3 a)}$ and $R_{\text {total }}^{(3 a)}$. The $G_{(\text {(generic) } \alpha \beta}^{(3 a)}$ is given by relation (16) and the Euler-Lagrange expressions of the $K_{9}$ to $K_{11}$ Lagrangian terms are given by relations (A.30)-(A.32). Also, we propose to find out the conditions for which $\operatorname{Trace} R_{(\text {total }) \alpha \beta}^{(3 a)}=R_{\text {total }}^{(3 a)}$.

After all the necessary substitutions and calculations, we find that the trace relation, for non-zero coefficients, holds if and only if

$$
\begin{array}{ll}
b_{5}=-60 b_{1}-122 b_{2} / 15-52 b_{3} / 15-b_{4} / 5, & b_{6}=30 b_{1}-4 b_{2} / 15-14 b_{3} / 15-17 b_{4} / 5 \\
b_{7}=30 b_{1}+32 b_{2} / 5+12 b_{3} / 5+13 b_{4} / 5, & b_{8}=100 b_{1}+40 b_{2} / 3+20 b_{3} / 3+2 b_{4} \\
b_{9}=b_{2} / 30-2 b_{3} / 15+b_{4} / 20, & b_{11}=-b_{10}=10 b_{9} .
\end{array}
$$

First of all, as it is evident from constraint (45), if any one of the $K_{9}$ or $K_{10}$ or $K_{11}$ Lagrangian terms is missing at the beginning (i.e., any one of the $b_{9}$ or $b_{10}$ or $b_{11}$ coefficients is zero), then the trace analogy will hold only if all of these terms vanish, and this case will return us to the case of part (a). This is confirmed once we set $b_{9}=0$ in constraint (45), whereby it reduces to constraint (28). Actually, we have checked this point straightforward by omitting one of the $K_{9}$ to $K_{11}$ terms just from the beginning. For example, by considering only the $K_{1}$ to $K_{10}$ terms, and carrying out the similar steps to what we have performed for the $K_{1}$ to $K_{11}$ terms, we find that the trace analogy imposes the constraint

$$
\begin{array}{lll}
b_{3}=0, & b_{4}=-2 b_{2} / 3, & b_{5}=-60 b_{1}-8 b_{2}, \\
b_{6}=30 b_{1}+2 b_{2}, & b_{7}=30 b_{1}+14 b_{2} / 3, & b_{8}=100 b_{1}+12 b_{2}, \\
b_{9}=0=b_{10}, & &
\end{array}
$$

which satisfies constraint (45). Indeed, it is constraint (28) when its $b_{3}$ coefficient vanishes, i.e. it is the corresponding case of (38) when the $b_{3}$ coefficient is zero instead of the $b_{8}$ one.

Also, one can easily get the same result by considering the outcomes of Ref. [19] in where they have also derived almost the dimensional dependent constraint version for the trace relation (through a proportionality parameter, say $u$ ) via classifying the six derivative Lagrangians of gravity whose the traced field equations have a reduced order (see the Appendix B). They have employed the $K_{1}$ to $K_{8}$

\footnotetext{
${ }^{18}$ We have dropped the prime sign on the $b_{i}$ 's.

${ }^{19}$ In this section, as we intend to employ the results only for the six-dimensional case, we apply the analogy only for the appearance of the $G_{(\text {total } \alpha \beta}^{(3 a)}$ in accord with part (a) of the previous section. However, we also perform the analogy for the appearance of the $G_{(\operatorname{total}) \alpha \beta}^{(3 b)}$, in accord with part (b), in Appendix B.
} 
terms plus $K_{15}, K_{16}, K_{17}$ (that by identities (A.24), (A.25) and (A.26) are equivalent to $K_{9}, K_{10}$ and $K_{11}$, respectively) and $K_{13}$ terms, even though they have also mentioned that these terms are not all linearly independent because of relations (A.20) and (A.22). Now, if one sets the coefficients of the $K_{11}$ and $K_{13}$ terms simultaneously zero in their result (their relation (B15)), then the coefficients of the $K_{9}$ and $K_{10}$ terms will vanish as well and the remaining relations (after substituting for their proportionality parameter in terms of the other coefficients, namely $\left.u=6(D-1) b_{1}+2 b_{2}\right)$ will reduce to constraint $(40) \cdot 20$

Yet, one may argue that identity (A.20) can equally be used for one of the other terms $K_{4}$ to $K_{8}$ as well. However, in such a case, if one substitutes for, e.g. and without lost of generality, the $K_{8}$ Lagrangian term, then the calculations will give constraint (45) when its $b_{8}$ coefficient is zero. Now, if one also sets $b_{9}=0$ in this new constraint, it will reduce to constraint (38) which is an especial case of part (a) in six dimensions. Therefore, in order to have a more general situation, it is more adequate to consider all the $K_{1}$ to $K_{11}$ terms simultaneously.21

Secondly, the trace analogy for the introduced $G_{(\text {total }) \alpha \beta}^{(3 a)}$ gives the number of independent $b_{i}$ 's to be four. This confirms an earlier analysis based on the cohomological point of view (see relation (4.13) of Ref. [17]) which also gives consistency conditions (again see the invariance condition (4.10) of Ref. [17] for numerical linear combinations of the first ten unknowns $b_{i}$ ). Also, the maximum number of permissible missing coefficients of $b_{1}$ to $b_{8}$ is three in constraint (45). However, once again in six dimensions, due to relation (37), one also has another extra constraint.

Let us now examine constraint (45) for the numerical coefficients of a few available Lagrangians. As mentioned in part (a), Deser et al. [13] (relation (25c) of their paper) and Karakhanyan et al. 37] (relation (2.18) of their paper) state that the corresponding Lagrangian density of the expression (after adapting the sign convention)

$$
A_{3} \equiv C^{\mu \nu \rho \tau} \square C_{\mu \nu \rho \tau}-2 C^{\mu \nu \rho \alpha} C_{\mu \nu \rho \beta} R_{\alpha}^{\beta}+3 C_{\mu \nu \rho \tau} R^{\mu \rho} R^{\nu \tau}+\frac{3}{2} K_{4}-\frac{27}{20} K_{2}+\frac{21}{100} K_{1}
$$

is also a Weyl-invariant combination in six dimension 22 After substituting for the Weyl tensor in six dimensions, it reads

$$
A_{3}=\frac{41}{100} K_{1}-\frac{15}{4} K_{2}+\frac{9}{2} K_{4}+5 K_{5}-2 K_{6}+\frac{1}{10} K_{9}-K_{10}+K_{11} .
$$

The coefficients of (48) satisfy constraint (45). Another similar expression is given in relation (4.7) of Ref. [17] as (after adapting the sign convention)

$$
M_{3} \equiv-K_{1}+8 K_{2}+2 K_{3}-10 K_{4}-10 K_{5}-\frac{1}{2} K_{9}+5 K_{10}-5 K_{11},
$$

that also satisfies constraint (45).

Karakhanyan et al. [37] also claim that there is an additiona 23 Weyl-invariant action in six dimensions with the scalar Lagrangian (relation (2.19) of their paper, after adapting the sign convention)

$$
A_{4} \equiv K_{10}-\frac{3}{10} K_{9}+2 C_{\mu \nu \rho \tau} R^{\mu \rho} R^{\nu \tau}-K_{4}+\frac{1}{10} K_{2}+\frac{1}{50} K_{1} .
$$

Again, after substituting for the Weyl tensor in six dimensions, it reads

$$
A_{4}=\frac{3}{25} K_{1}-K_{2}+2 K_{5}-\frac{3}{10} K_{9}+K_{10} .
$$

\footnotetext{
${ }^{20}$ Note that, if one sets only the coefficient of the $K_{13}$ term zero and $D=6$ in their result, and also substitutes for their proportional parameter in terms of the other coefficients, one will get constraint (45).

${ }^{21}$ The same third order terms (though in different combinations) have also been used in Ref. 37] (its relations (2.12)(2.17)) as all six-dimensional dimensionless actions.

${ }^{22}$ For the Weyl-invariant expressions in arbitrary dimensions, see Refs. [19, 33, 38.

${ }^{23}$ However, the relations $A_{3}$ and $A_{4}$ differ 37 from $A_{1}$ and $A_{2}$ in that they have non-zero Weyl variations and one can employ them (only) as constraints on local counterterms, but those cannot be considered as independent contributions into the anomaly.
} 
Now, if one properly inserts the $K_{11}$ term from identity (A.20) into relation (51), then it will read effectively a:24

$$
A_{4} \stackrel{\text { eff }}{=} \frac{3}{25} K_{1}-K_{2}+\frac{4}{3} K_{4}+\frac{2}{3} K_{5}-\frac{2}{3} K_{6}+\frac{2}{3} K_{7}+\frac{4}{3} K_{8}+\frac{1}{30} K_{9}-\frac{1}{3} K_{10}+\frac{1}{3} K_{11},
$$

that its coefficients satisfy constraint (45).

Arakelyan et al. [39] give in relation (18) of their preprint, as the third linear cocycle, the expression (after adapting the sign convention)

$$
\begin{aligned}
S_{C}^{3} \equiv \int[ & C^{\alpha \beta \mu \nu} \square C_{\alpha \beta \mu \nu}+4 C^{\alpha \mu \nu \rho} C_{\beta \mu \nu \rho} R_{\alpha}^{\beta}-\frac{6}{5} C^{\alpha \mu \nu \rho} C_{\alpha \mu \nu \rho} R \\
& \left.+8\left(C^{\alpha \mu \nu \rho} C_{\beta \mu \nu \rho}\right)^{; \beta}{ }_{\alpha}-\frac{1}{2} \square\left(C^{\alpha \mu \nu \rho} C_{\alpha \mu \nu \rho}\right)\right] \sqrt{-g} d^{6} x \equiv \int L_{C}^{3} \sqrt{-g} d^{6} x .
\end{aligned}
$$

Substituting for the Weyl tensor in six dimensions, the used Lagrangian, apart from the complete divergent terms, is actually

$$
L_{C}^{3} \stackrel{\text { eff }}{=}-\frac{11}{50} K_{1}+\frac{27}{10} K_{2}-\frac{6}{5} K_{3}-3 K_{4}-4 K_{5}+4 K_{6}+\frac{1}{10} K_{9}-K_{10}+K_{11},
$$

that also satisfies constraint (45). Another similar conformal anomaly is given in relation (5) of Ref. [40] as (after adapting the sign convention and correcting a minor mistype)

$$
\begin{aligned}
T_{A} \equiv \frac{1}{540 \times(4 \pi)^{3}} \int[ & -\frac{1}{300} K_{1}+\frac{1}{10} K_{2}-\frac{1}{10} K_{3}-\frac{3}{7} K_{4}+\frac{5}{21} K_{5}-\frac{2}{21} K_{6}+\frac{2}{7} K_{7}-\frac{23}{210} K_{9} \\
& \left.+\frac{13}{42} K_{10}+\frac{1}{12} K_{11}\right] \sqrt{-g} d^{6} x \equiv \frac{1}{540 \times(4 \pi)^{3}} \int L_{A} \sqrt{-g} d^{6} x,
\end{aligned}
$$

where, by properly inserting the $K_{11}$ term from identity (A.20), the Lagrangian effectively reads

$$
L_{A} \stackrel{\text { eff }}{=}-\frac{1}{300} K_{1}+\frac{1}{10} K_{2}-\frac{1}{10} K_{3}+\frac{2}{21} K_{4}-\frac{2}{7} K_{5}-\frac{5}{14} K_{6}+\frac{23}{42} K_{7}+\frac{11}{21} K_{8}+\frac{3}{140} K_{9}-\frac{3}{14} K_{10}+\frac{3}{14} K_{11} .
$$

These coefficients satisfy constraint (45).

Also, two Weyl-invariants (denoted by $\Sigma$ and $\Theta$ ) have been introduced in arbitrary dimensions in Ref. [19] (their relations (B17) and (B18)), which in six dimensions are

$$
\begin{aligned}
\left.\Theta\right|_{6-\operatorname{dim} .} & =-\left.8 \Sigma\right|_{6-\operatorname{dim} .} \\
& \equiv-8\left(-\frac{4}{25} K_{1}+2 K_{4}+6 K_{5}-8 K_{7}-16 K_{8}-\frac{9}{10} K_{15}+3 K_{16}+\text { total derivative }\right) .
\end{aligned}
$$

Using identities (A.24) and (A.25), and again properly inserting the $K_{11}$ term from identity (A.20), the conformal anomaly (57) effectively reads

$$
\left.\Sigma\right|_{6-\text { dim. }} \stackrel{\text { eff }}{=}-\frac{4}{25} K_{1}-2 K_{4}+10 K_{5}+2 K_{6}-10 K_{7}-20 K_{8}-\frac{1}{10} K_{9}+K_{10}-K_{11},
$$

that its coefficients satisfy constraint (45).

In the next section, we examine the outcome of our rigorous pursuit of the trace analogy approach for the leaded trace anomaly (which also indulges into the heat kernel) from the third order terms in six dimensions.

\footnotetext{
${ }^{24}$ Indeed, relation (51) should be the effective one.
} 


\section{Classical Trace Anomaly}

As mentioned in the Introduction, by enforcing the mathematical appearance of the alternative form of the Einstein field equations, i.e. $R_{\alpha \beta}=\kappa^{2} S_{\alpha \beta} / 2$ where the source tensor is $S_{\alpha \beta} \equiv T_{\alpha \beta}-$ $g_{\alpha \beta} T /(D-2)$ in a $D$-dimensional space-time with $T=\operatorname{Trace} T_{\alpha \beta}$, for the relevant alternative form of the Lovelock field equations, we have classically justified [3] that one gets

$$
R_{\alpha \beta}^{(\text {Lovelock) }}=\frac{1}{2} \kappa^{2} S_{\alpha \beta}^{\text {(genera.) }},
$$

where the generalized source tensor is

$$
S_{\alpha \beta}^{\text {(genera.) }} \equiv T_{\alpha \beta}-\frac{1}{D-2} g_{\alpha \beta}\left(T+T_{\text {anomaly }}\right)
$$

and

$$
T_{\text {anomaly }}=-\kappa^{-2} D \sum_{n \geq 1}^{n_{\text {ext. }}} \frac{n-1}{n} c_{n} R^{(n)} \equiv \sum_{n \geq 1}^{n_{\text {ext. }}} T_{\text {anomaly }}^{(n)} .
$$

This shows that $T_{\text {anomaly }}^{(1)}=0$, as expected to be for the Einstein gravity, and gives the dimension of the $c_{n}$ to be length to the power of $2(n-1)$, as indicated below the Lovelock Lagrangian (3).

Then, we have applied [3] the outcome for the generic cases, i.e. $R_{\text {generic }}^{(n)}$, with the appropriate constraint condition on the coefficients and no upper limit for $n$. Actually, we have examined only the resulted second order trace anomaly in Ref. [3. That is

$$
T_{\text {anomaly }}^{(2)}=-\frac{\kappa^{-2} D}{2} c_{2} R_{\text {generic }}^{(2)},
$$

where [3, 10]

$$
R_{\text {generic }}^{(2)} \equiv \kappa^{2} L_{\text {generic }}^{(2)}-\left(4 a_{1}+a_{2}\right) \square R=a_{1} R^{2}+a_{2} R_{\mu \nu} R^{\mu \nu}+a_{3} R_{\alpha \beta \mu \nu} R^{\alpha \beta \mu \nu}-\left(4 a_{1}+a_{2}\right) \square R,
$$

with the constraint

$$
3 a_{1}+a_{2}+a_{3}=0
$$

that leaves two degrees of freedom in $D>4$ dimensions, or with the constraint, e.g., $\alpha_{2}+\alpha_{3}=0$ with one degree of freedom in (and up to) four dimensions (due to the Gauss-Bonnet term), where $\alpha_{2}$ and $\alpha_{3}$ are just the new coefficients for the corresponding terms.

Now in this work, we investigate the third order trace anomaly resulted from the introduced total third order Lagrangian (43). The trace anomaly issue for this case is

$$
T_{\text {anomaly }}^{(3)}=-\frac{2 \kappa^{-2} D}{3} c_{3} R_{\text {total }}^{(3 a)},
$$

with constraint (45) and, as defined in (44),

$$
R_{\text {total }}^{(3 a)} \equiv \kappa^{2} L_{\text {generic }}^{(3)}+M^{(3)}+b_{9} R^{\left(K_{9}\right)}+b_{10} R^{\left(K_{10}\right)}+b_{11} R^{\left(K_{11}\right)} .
$$

The explicit expression of (66), after substituting from (14), (19), (A.30), (A.31) and (A.32) when considering constraint (45), is

$$
\begin{aligned}
R_{\text {total }}^{(3 a)}= & b_{1} K_{1}+b_{2} K_{2}+b_{3} K_{3}-\left(120 b_{1}+\frac{244}{15} b_{2}+\frac{104}{15} b_{3}+\frac{12}{5} b_{4}\right) K_{4} \\
& +\left(60 b_{1}+\frac{122}{15} b_{2}+\frac{52}{15} b_{3}+\frac{16}{5} b_{4}\right) K_{5}-\left(8 b_{2}+8 b_{3}+6 b_{4}\right) K_{6} \\
& +\left(60 b_{1}+\frac{212}{15} b_{2}+\frac{142}{15} b_{3}+\frac{26}{5} b_{4}\right) K_{7}+\left(160 b_{1}+\frac{144}{5} b_{2}+\frac{104}{5} b_{3}\right.
\end{aligned}
$$




$$
\begin{aligned}
& \left.+\frac{36}{5} b_{4}\right) K_{8}-\left(12 b_{1}+b_{2}\right) K_{9}+\left(120 b_{1}+\frac{58}{5} b_{2}+\frac{48}{5} b_{3}-\frac{3}{5} b_{4}\right) K_{10} \\
& +\left(4 b_{2}+3 b_{4}\right) K_{11}-\left(60 b_{1}+\frac{152}{15} b_{2}+\frac{52}{15} b_{3}+\frac{16}{5} b_{4}\right) K_{12} \\
& -\left(180 b_{1}+\frac{476}{15} b_{2}+\frac{316}{15} b_{3}+\frac{43}{5} b_{4}\right) K_{13}+\left(\frac{1}{5} b_{2}-\frac{4}{5} b_{3}+\frac{3}{10} b_{4}\right) K_{14} \\
& +\left(-12 b_{1}-\frac{61}{30} b_{2}+\frac{2}{15} b_{3}-\frac{4}{5} b_{4}\right) K_{15}+\left(180 b_{1}+\frac{137}{5} b_{2}+\frac{112}{5} b_{3}\right. \\
& \left.+\frac{51}{10} b_{4}\right) K_{16}+\left(-15 b_{1}-\frac{1}{5} b_{2}-\frac{11}{5} b_{3}+\frac{6}{5} b_{4}\right) K_{17},
\end{aligned}
$$

with four degrees of freedom in $D>6$ dimensions. By considering identities (A.21), (A.22) and (A.24) - (A.26), it effectively reads (though, we have purposely kept the complete divergent term $K_{14}$ )

$$
\begin{aligned}
R_{\text {total }}^{(3 a) \stackrel{\text { eff }}{=}} & b_{1} K_{1}+b_{2} K_{2}+b_{3} K_{3}+\left(60 b_{1}+\frac{232}{15} b_{2}+\frac{212}{15} b_{3}+\frac{31}{5} b_{4}\right) K_{4} \\
& -\left(120 b_{1}+\frac{118}{5} b_{2}+\frac{88}{5} b_{3}+\frac{27}{5} b_{4}\right) K_{5}-\left(8 b_{2}+8 b_{3}+6 b_{4}\right) K_{6} \\
& +\left(60 b_{1}+\frac{212}{15} b_{2}+\frac{142}{15} b_{3}+\frac{26}{5} b_{4}\right) K_{7}+\left(160 b_{1}+\frac{144}{5} b_{2}+\frac{104}{5} b_{3}+\frac{36}{5} b_{4}\right) K_{8} \\
& +\left(15 b_{1}+\frac{39}{10} b_{2}+\frac{17}{5} b_{3}+\frac{27}{20} b_{4}\right) K_{9}-\left(60 b_{1}+\frac{79}{5} b_{2}+\frac{64}{5} b_{3}+\frac{57}{10} b_{4}\right) K_{10} \\
& +\left(15 b_{1}+\frac{21}{5} b_{2}+\frac{11}{5} b_{3}+\frac{9}{5} b_{4}\right) K_{11}+\left(\frac{1}{5} b_{2}-\frac{4}{5} b_{3}+\frac{3}{10} b_{4}\right) K_{14} .
\end{aligned}
$$

On the other hand, in the quantum aspects of gravity, it has been shown that for $D=6(=2 \times 3)$ dimensions, the finite and renormalized expectation value of the trace of the energy-momentum tensor, $\left\langle T_{\rho}{ }^{\rho}\right\rangle_{\text {ren }}$, would have to be [4] cubic in curvature, and so on for $D=2 n$ dimensions to be to the $n$th power in curvature 25 This effect is despite the fact that the classical energy-momentum tensors, for the conformally invariant classical actions, must be traceless. And, it is known as a conformal, or trace, or Weyl anomaly, originally noticed in 1973 [41], which plays an important role in understanding of many phenomena [4, 5, 42. Actually, the anomalies generally occur in any regularization method as a consequence of introducing a scale into the theory in order to regularize it, see, e.g., Ref. 8]. The contribution of a divergent Lagrangian to the trace of the energy-momentum tensor is one of the above consequences. Of course, when the effective action is itself a conformally invariant action, the expectation value of the trace of the total energy-momentum tensor is zero.

We have indicated [3] that constraint (64) is exactly the same as the consistency condition on the numerical coefficients that Duff suggested [12] in the process of re-examining the Weyl anomaly applications when the dimensional regularization is applied to a classically conformally invariant theory in arbitrary dimension. Also, relation (62) gives exactly the same result as in Ref. [43], in where it has been shown that the relevant most general form of the anomalous trace of the energymomentum tensor for classically conformally invariant fields of arbitrary spin and dimension is

$$
\left\langle T_{\rho}^{\rho}\right\rangle_{\mathrm{ren}}=-\frac{\hbar c}{180(4 \pi)^{2}}\left(a_{1} R^{2}+a_{2} R_{\mu \nu} R^{\mu \nu}+a_{3} R_{\alpha \beta \mu \nu} R^{\alpha \beta \mu \nu}+\gamma \square R\right) .
$$

By comparison, it obviously shows that $\gamma=-\left(4 a_{1}+a_{2}\right)$, which completes the trace anomaly relations suggested by Duff [12] and, in four dimensions, reveals $c_{2} \propto \ell_{P}{ }^{2}$, as expected.

The trace anomalies are [4 precisely the $\mathbf{b}_{\mathbf{m}}$ coefficients [also referred to as Hamidew (after Hadamard-Minakshisundaram-DeWitt) 44] or, HMDS (after the same persons plus Seely) [45] or, Minakshisundaram-Pleijel [46] coefficients] of the Schwinger-DeWitt proper time method, see, e.g., Ref. [45] and references therein. These are the t-independent terms in the asymptotic expansion of

\footnotetext{
${ }^{25}$ For a brief review of this subject see, e.g., Ref. [3] and references therein.
} 
the heat kerne 26 with the appropriate differential operator $\triangle$, see, e.g., Refs. [34, 47], in

$$
\text { trace } e^{-\triangle t} \sim \sum_{\substack{m=0 \\ \text { even no. }}}^{\infty} B_{m} t^{\frac{m-D}{2}} \quad t \rightarrow 0^{+},
$$

where $B_{m}=\int \mathbf{b}_{\mathbf{m}} \sqrt{-g} d^{D} x$ are invariants of the differential operator and vanish for odd numbers of the $m$. The calculation of the first order of these coefficients by the pioneering method of DeWitt [48] is quite simple, but gets very cumbersome at higher orders. Indeed, due to the combinatorial explosion in the number of terms in the $\mathbf{b}_{\mathbf{m}}$ and in the auxiliary tensorial quantities, improvement in the higher orders $m$ has been tedious. Though, new algorithms and computer algebra with improvements in computer systems have appeared to perform great efficiency, see, e.g., Refs. [27, 49] and references therein.

As in the literature, there is not, up to now, an explicit calculated expression for the trace anomaly in six dimensions in the semi-classical theory, we examine our third order trace anomaly result (65) by a straightforward use of the $\mathbf{b}_{6}$ coefficient of the Schwinger-DeWitt proper time method. Though, let us first apply this comparison, as a re-examining, for the second order one with the $\mathbf{b}_{\mathbf{4}}$ coefficient.

For this purpose, if the $\triangle$ in $(\sqrt{70})$ being the simplest of such an operator, e.g., the conformally invariant Laplacian type operator 27

$$
\triangle=\square-\xi(D) R
$$

then the $\mathbf{b}_{\mathbf{4}}$, when the conformal coupling constant is $\xi(4)=1 / 6$, will be given by 28

$$
\mathbf{b}_{4}=-\frac{\hbar c}{180(4 \pi)^{2}}\left(R_{\mu \nu} R^{\mu \nu}-R_{\alpha \beta \mu \nu} R^{\alpha \beta \mu \nu}-\square R\right)
$$

which is the trace anomaly in the case of massless conformal scalar fields in four dimensions. On the other hand, the trace anomaly (62), by substituting (63) with constraint (64), gives

$$
T_{\text {anomaly }}^{(2)}=-\frac{\kappa^{-2} D}{2} c_{2}\left[-\frac{1}{3}\left(a_{2}+a_{3}\right) R^{2}+a_{2} R_{\mu \nu} R^{\mu \nu}+a_{3} R_{\alpha \beta \mu \nu} R^{\alpha \beta \mu \nu}+\frac{1}{3}\left(a_{2}+4 a_{3}\right) \square R\right],
$$

that, using the Gauss-Bonnet theorem [35] in four dimensions, effectively reads

$$
T_{\text {anomaly }}^{(2)} \stackrel{\text { eff }}{=}-2 \kappa^{-2} c_{2}\left[-\frac{1}{3}\left(a_{2}+4 a_{3}\right)\right]\left(R_{\mu \nu} R^{\mu \nu}-R_{\alpha \beta \mu \nu} R^{\alpha \beta \mu \nu}-\square R\right) .
$$

In comparison with (72), this consistently gives the constraint $a_{2}+4 a_{3}=-3$ (or, using (64), equivalently $4 a_{1}+a_{2}=1$ ) with one degree of freedom and the same numerical value for $c_{2}$, as expected.

Now, let us investigate the issue for the third order trace anomaly. By using the relation $E_{3}$ of the theorem 4.3 of Ref. [51], when its $\mathcal{E}=\xi(D) R$, the conformal coupling constant is $\xi(6)=1 / 5$, the $W_{i j}=0$, a minor mistyped is corrected 29 and adapting the sign convention, one gets

$$
\begin{aligned}
\mathbf{b}_{6}=-\frac{1}{360(4 \pi)^{3}}( & \frac{1}{450} K_{1}-\frac{1}{15} K_{2}+\frac{1}{15} K_{3}-\frac{4}{63} K_{4}+\frac{4}{21} K_{5}+\frac{8}{21} K_{6} \\
& -\frac{32}{63} K_{7}-\frac{40}{63} K_{8}+\frac{4}{7} K_{10}-\frac{6}{7} K_{11}-\frac{2}{35} K_{12}+\frac{2}{7} K_{13} \\
& \left.-\frac{3}{35} K_{14}-\frac{1}{70} K_{15}+\frac{1}{7} K_{16}-\frac{9}{14} K_{17}\right) .
\end{aligned}
$$

This relation gives the trace anomaly in the case of massless conformal scalar fields in six dimension 30 The same issue has also been worked out by using an alternative method in Ref. [46], in where from

\footnotetext{
${ }^{26}$ It is a very powerful tool in the mathematical physics as well as in the quantum field theory.

${ }^{27}$ We have checked the signs with Ref. [50, and $\xi(D)=(D-2) /[4(D-1)]$.

${ }^{28} \mathrm{See}$, e.g., the relation $E_{4}$ of Ref. [47] when its $E=R / 6$ and $W_{i j}=0$, and also adapting the sign convention.

${ }^{29}$ Its term $-4 R_{i j i k} R_{; j k}$ must read $-4 R_{i j i k} \mathcal{E}_{; j k}$, see also Ref. 52 .

${ }^{30}$ The coefficient $-1 /\left[360(4 \pi)^{3}\right]$ is given in the natural units, otherwise it reads $-\hbar^{2} G /\left[360(4 \pi)^{3} c^{2}\right]$, for making the dimension of the $\mathbf{b}_{6}$ coefficient to be the same as the trace anomaly.
} 
their relation (3.3) with $\xi(6)=1 / 5$ and using relation (12), one achieves

$$
\begin{aligned}
\mathbf{b}_{6}=-\frac{1}{360(4 \pi)^{3}}( & \frac{1}{450} K_{1}-\frac{1}{15} K_{2}+\frac{1}{15} K_{3}-\frac{4}{63} K_{4}+\frac{4}{21} K_{5}-\frac{4}{3} K_{6} \\
& +\frac{76}{63} K_{7}+\frac{176}{63} K_{8}+\frac{4}{7} K_{10}-\frac{2}{35} K_{12}+\frac{2}{7} K_{13}-\frac{3}{35} K_{14} \\
& \left.-\frac{1}{70} K_{15}+\frac{1}{7} K_{16}-\frac{9}{14} K_{17}+\frac{24}{7} R_{\mu \nu ; \lambda \rho} R^{\lambda \mu \nu \rho}\right)
\end{aligned}
$$

which is exactly the same as relation (75) once the last relation of (A.1) is substituted. However, by considering identities (A.21), (A.22) and (A.24)-(A.26), the trace anomaly relation (75) effectively reads

$$
\begin{aligned}
\mathbf{b}_{6} \stackrel{\text { eff }}{=}-\frac{1}{360(4 \pi)^{3}} & \left(\frac{1}{450} K_{1}-\frac{1}{15} K_{2}+\frac{1}{15} K_{3}-\frac{22}{63} K_{4}+\frac{10}{21} K_{5}+\frac{8}{21} K_{6}\right. \\
& \left.-\frac{32}{63} K_{7}-\frac{40}{63} K_{8}-\frac{3}{35} K_{9}+\frac{3}{7} K_{10}-\frac{3}{14} K_{11}-\frac{3}{35} K_{14}\right) .
\end{aligned}
$$

On the one hand, by setting the coefficients $b_{1}=1 / 450, b_{2}=-1 / 15, b_{3}=1 / 15$ and $31 b_{4}=-4 / 63$ in our relation (68), we also get in six dimensions (though, we have not used identity (37) yet),

$$
\begin{aligned}
T_{\text {anomaly }}^{(3)} \stackrel{\text { eff }}{=}-4 \kappa^{-2} c_{3}( & \frac{1}{450} K_{1}-\frac{1}{15} K_{2}+\frac{1}{15} K_{3}-\frac{22}{63} K_{4}+\frac{10}{21} K_{5}+\frac{8}{21} K_{6} \\
& \left.-\frac{32}{63} K_{7}-\frac{40}{63} K_{8}-\frac{3}{35} K_{9}+\frac{3}{7} K_{10}-\frac{3}{14} K_{11}-\frac{3}{35} K_{14}\right) .
\end{aligned}
$$

As it is evident, our result (78) is exactly the same as relation (77), and by comparison we also have $c_{3} \propto \ell_{P}^{4}$, as expected.

\section{Conclusions}

In our previous works, we have shown that the analogy of the Einstein tensor splitting into two parts with the trace relation between them can be performed not only for each separate (homogeneous) term of the Lovelock tensor, but also for the (whole) Lovelock tensor as a complete Lagrangian (and indeed, for any inhomogeneous Euler-Lagrange expression that can be spanned linearly in terms of homogeneous tensors), via a generalized trace operator, as well [1]. For the second term of the Lovelock tensor, we have discovered that it is not only this term, treated as an Euler-Lagrange expression of a special combination of the second order Lagrangian terms, that possesses this analogy and satisfies the trace relation, but also there are the Euler-Lagrange expressions of the other generic cases of the second order Lagrangian terms whose constant coefficients satisfy a specific constraint, i.e. either exactly the Duff trace anomaly relation or a dimensional dependent version of it [3, 10]. We have extended [3] the analogy further, and have manifested that the analogy of the alternative form of the Einstein field equations for the relevant alternative form of the Lovelock field equations reveals a classical approach toward the trace anomaly with an indication of the constitution of the higher order gravities towards it. Indeed, we have explicitly shown 3 , that this procedure for any generic coefficients of the second order term of the Lovelock Lagrangian yields exactly the Duff trace anomaly relation, and even have achieved [10] a dimensional dependent version of this relation.

In this work, we have probed further the analogy for the generic coefficients of the eight terms in the third order of the Lovelock Lagrangian, and have found the constraint relations between the nonzero constituent coefficients into two forms, an independent and a dimensional dependent versions. Each form has three degrees of freedom, and the dimensional dependent constraint in six dimensions is exactly the same as the other one. They do not allow simultaneously the missing of more than

\footnotetext{
${ }^{31}$ Note that, $b_{1}, b_{2}$ and $b_{3}$ are evident from relation (77) when are compared with relation (68), and $b_{4}$ can be found from the coefficient of the term $K_{6}$ when one matches these relations in the case of $b_{2}=-1 / 15=-b_{3}$.
} 
three coefficients. The coefficients of the third order term of the Lovelock Lagrangian do satisfy the two forms of the constraints, where in particular they yield the dimensional dependent one in any dimension. The two independent Lagrangian densities made from the cubic of the Weyl tensor (as conformal invariants in six dimensions) also satisfy the independent constraint only in six dimensions, and yield the dimensional dependent version identically independent of the dimension.

We have specified the all seventeen independent terms of the third order type Lagrangian with the HDN three. Among these terms, we have justified (by using a few complete divergent terms that lead to the relevant identities) and have introduced the most general effective expression as a total third order type Lagrangian with arbitrary coefficients of just the eleven terms of them (the previous eight terms plus the new three ones) denoted as the $K_{1}$ to $K_{11}$ in the text. Then, we have proceeded the analogy for the field tensor of this combination, and have achieved the relevant constraint among the non-zero constituent coefficients. The constraint shows that, if one of these three new Lagrangian terms is missed, then all of them will vanish (whereby the constraint reduces to the previous case). Also, the maximum number of permissible missing coefficients of the first previous eight ones is again three. There are, in general, four degrees of freedom, though in six dimensions, there exists another extra identity among the first eight coefficients. We have shown that the expressions given in the literature as the third Weyl-invariant combination in six dimensions do satisfy the obtained constraint relations. Thus, we suggest that these constraint relations to be considered as the necessary consistency conditions on the numerical coefficients that a Weyl-invariant should satisfy (similar to the Duff consistency relations for the second order trace anomaly).

We have reviewed the "classical" approach toward the trace anomaly that was presented in our previous works, in order to examine it for the introduced total third order type Lagrangian, by then, we have derived the relevant trace anomaly. To examine our outcome, as an explicit calculated expression for the trace anomaly in six dimensions in the semi-classical theory has not been given in the literature, we have compared our result with the precisely equivalent expression, namely the $\mathbf{b}_{\mathbf{6}}$ coefficient of the Schwinger-DeWitt proper time method that linked with the relevant heat kernel coefficient. For this purpose, we first have achieved our general expressions for the trace anomaly for the generic second order (as a re-examining case) and then, for the total third order types Lagrangian terms, relations (73) and (68), with two and four degrees of freedom (in $D>6$ dimensions, and three in six dimensions), respectively. Then, we have demonstrated that the obtained expressions contain exactly the $\mathbf{b}_{\mathbf{4}}$ and $\mathbf{b}_{\mathbf{6}}$ coefficients, respectively, as a particular case. Of course, these results are necessary consistency checks, nevertheless our approach can be regarded as an alternative (perhaps simpler, and classical) derivation of the trace anomaly which also gives a general expression with the relevant degrees of freedom.

In aside, let us assert our view about the approach employed in the work for general cases. The results obtained indicate that it is likely that the analogy of the Einstein gravity should also exist for a class of further generic Lagrangians of order/degree $n>6$. However, for each order/degree to hold the analogy, there would be a set of constraints that the relevant constituent coefficients must satisfy. Of course, extending the analogy to higher orders of generic cases does not seem technically very easy (even in the third order case considerable algebraic calculations was required). That is, in order to find the constraints for generic cases, or for the most general effective expressions as total Lagrangian terms of each order, work must be performed order by order with huge calculations for each one.

\section{Acknowledgements}

We thank the Research Office of Shahid Beheshti University G.C. for financial supporting of this work. 


\section{Appendix A: Useful Relations \& Variation Of A Few Actions}

In this appendix, we furnish a few useful relations and also supply the metric variation of a few Lagrangian terms.

\section{- The Useful Relations}

The following derivative relations can easily be derived from non-commutativity of the covariant derivatives, and the Bianchi and the contracted Bianchi identities, as [26]

$$
\begin{aligned}
& R_{\alpha \mu}{ }^{; \mu}{ }_{\beta}=R_{; \alpha \beta} / 2, \\
& R_{\mu \alpha \nu \beta}{ }^{; \alpha}=R_{\mu \nu ; \beta}-R_{\mu \beta ; \nu}, \\
& R_{\mu \tau ; \nu}{ }^{\alpha}=R_{; \mu \nu} / 2-R_{\mu \alpha \nu \beta} R^{\alpha \beta}+R_{\mu \alpha} R_{\nu}{ }^{\alpha} \\
& R_{\nu \tau \mu \rho} R^{\tau \mu ; \nu \rho}=K_{6} / 2-K_{7} / 2-K_{8}-K_{11} / 4
\end{aligned}
$$

and also

$$
\left(R^{k-1}\right)_{; \mu \nu}=(k-1) R^{k-2} R_{; \mu \nu}+(k-1)(k-2) R^{k-3} R_{; \mu} R_{; \nu} .
$$

In the variation process, using the integrating covariantly by parts and the appropriate boundary conditions, one can effectively write

$$
f \delta\left(\square^{k} R\right) \stackrel{\text { eff }}{=}\left(\square^{k} f\right) \delta R+\sum_{i=0}^{k-1}\left(\square^{i} f\right)\left[-\left(\square^{(k-1-i)} R\right)_{, \tau} g^{\alpha \rho} \delta \Gamma^{\tau} \alpha \rho+\left(\square^{(k-1-i)} R\right)_{; \alpha \rho} \delta g^{\alpha \rho}\right],
$$

where the $f$ is any scalar function of the metric and its derivatives.

The Euler-Lagrange expressions of a few scalar Lagrangian density term 32 where $\mathcal{L}=F \sqrt{-g} / \kappa^{2}$, are [26]

$$
\begin{aligned}
& G_{\alpha \beta}^{(F(R))}=F^{\prime} R_{\alpha \beta}-F^{\prime} ; \alpha \beta-\frac{1}{2} g_{\alpha \beta}\left(F-2 \square F^{\prime}\right), \\
& G_{\alpha \beta}^{\left(F\left(R_{\mu \nu} R^{\mu \nu}\right)\right)}=2 F^{\prime} R_{\alpha \rho} R_{\beta}{ }^{\rho}-\left(F^{\prime} R_{\alpha \rho}\right)_{; \beta}{ }^{\rho}-\left(F^{\prime} R_{\beta \rho}\right)_{; \alpha}{ }^{\rho}+\square\left(F^{\prime} R_{\alpha \beta}\right) \\
& -\frac{1}{2} g_{\alpha \beta}\left[F-2\left(F^{\prime} R^{\mu \nu}\right)_{; \mu \nu}\right] \text {, } \\
& G_{\alpha \beta}^{\left(F\left(R_{\rho \tau \mu \nu} R^{\rho \tau \mu \nu}\right)\right)}=2 F^{\prime} R_{\alpha \rho \mu \nu} R_{\beta}^{\rho \mu \nu}+2\left(F^{\prime} R_{\mu \alpha \nu \beta}\right)^{; \mu \nu}+2\left(F^{\prime} R_{\mu \beta \nu \alpha}\right)^{; \mu \nu}-\frac{1}{2} g_{\alpha \beta} F \text {, } \\
& \left.G_{\alpha \beta}^{\left(F\left(\sum_{k=0}^{p} \square^{k} R\right)\right)}=\left(\sum_{k=0}^{p} \square^{k} f_{k}\right) R_{\alpha \beta}-\left(\sum_{k=0}^{p} \square^{k} f_{k}\right)_{; \alpha \beta}-\sum_{k=1}^{p} \sum_{i=0}^{k-1}\left(\square^{i} f_{k}\right)_{(; \alpha}\left(\square^{(k-1-i)} R\right)_{; \beta}\right) \\
& -\frac{1}{2} g_{\alpha \beta}\left\{F-2 \square\left(\sum_{k=0}^{p} \square^{k} f_{k}\right)-\sum_{k=1}^{p} \sum_{i=0}^{k-1}\left[\left(\square^{i} f_{k}\right)\left(\square^{(k-1-i)} R\right)_{; \rho}\right]^{; \rho}\right\}
\end{aligned}
$$

and

$$
\begin{aligned}
G_{\alpha \beta}^{\left(R^{N} \square^{l} R\right)=} & \Theta^{[N l]} R_{\alpha \beta}-\Theta_{; \alpha \beta}^{[N l]}-\sum_{i=0}^{l-1}\left(\square^{i} R^{N}\right)_{(; \alpha}\left(\square^{(l-1-i)} R\right)_{; \beta)} \\
& -\frac{1}{2} g_{\alpha \beta}\left\{R^{N} \square^{l} R-2 \square \Theta^{[N l]}-\sum_{i=0}^{l-1}\left[\left(\square^{i} R^{N}\right)\left(\square^{(l-1-i)} R\right)_{; \rho}\right]\right\}
\end{aligned}
$$

where the prime denotes ordinary derivative with respect to the argument, $f_{k} \equiv \partial F / \partial \square^{k} R$ and $\Theta^{[N l]} \equiv N R^{N-1} \square^{l} R+\square^{l} R^{N}$.

\footnotetext{
${ }^{32}$ The corresponding Euler-Lagrange expressions of Lagrangians containing the derivatives of the curvature scalar are firstly due to Buchdahl $[53$.
} 
The Weyl conformal tensor, for $D \geq 3$ dimensions, satisfies

$$
C_{\alpha \beta \mu \nu} C^{\alpha \beta \mu \nu}=\frac{2}{(D-1)(D-2)} R^{2}-\frac{4}{(D-2)} R_{\mu \nu} R^{\mu \nu}+R_{\alpha \beta \mu \nu} R^{\alpha \beta \mu \nu},
$$

where in four dimensions $\left.C_{\alpha \beta \mu \nu} C^{\alpha \beta \mu \nu}\right|_{4-\text { dim. }}=R^{2} / 3-2 R_{\mu \nu} R^{\mu \nu}+R_{\alpha \beta \mu \nu} R^{\alpha \beta \mu \nu}$. And, its associated effective Lagrangian, by considering the Gauss-Bonnet theorem, is $-2 / 3\left(R^{2}-3 R_{\mu \nu} R^{\mu \nu}\right)$. In six dimensions, it reads $\left.C_{\alpha \beta \mu \nu} C^{\alpha \beta \mu \nu}\right|_{6 \text {-dim. }}=R^{2} / 10-R_{\mu \nu} R^{\mu \nu}+R_{\alpha \beta \mu \nu} R^{\alpha \beta \mu \nu}$, also $\left.C_{\alpha \beta \mu \nu} \square C^{\alpha \beta \mu \nu}\right|_{6 \text {-dim. }}=$ $K_{9} / 10-K_{10}+K_{11}$ and $\left.C_{\alpha \beta \mu \nu ; \rho} C^{\alpha \beta \mu \nu ; \rho}\right|_{6-\operatorname{dim} .}=K_{15} / 10-K_{16}+K_{17}$. In three dimensions, as the Weyl tensor identically vanishes, from relations (32) and (33) we have, respectively,

$$
\left.\left(6 K_{1}-36 K_{2}+3 K_{3}+32 K_{4}+24 K_{5}-12 K_{6}+K_{7}\right)\right|_{3-\operatorname{dim} .} \equiv 0
$$

and

$$
\left.\left(-7 K_{1} / 2+21 K_{2}-3 K_{3} / 2-18 K_{4}-15 K_{5}+6 K_{6}+K_{8}\right)\right|_{3-\operatorname{dim} .} \equiv 0 .
$$

In four dimensions, from relations (32) and (33) and their dependence, one has

$$
\left.\left(\frac{8}{9} K_{1}+18 K_{2}-3 K_{3}-20 K_{4}-24 K_{5}+18 K_{6}-K_{7}+4 K_{8}\right)\right|_{4-\operatorname{dim} .} \equiv 0 .
$$

Also, see Ref. [33] and the appendix of Ref. [34]. The scalar action $I$ constructed by the cubic of the Weyl tensor, relations (32) or (33), in a $D$-dimensional space-time, through the conformal transformation $g_{\mu \nu} \rightarrow \Omega^{2} g_{\mu \nu}$, conformally transforms as $I \rightarrow \Omega^{D-6} I$. Hence, it is a conformal invariant only in six dimensions.

\section{- The $M^{(3)}$ As A Lagrangian Term}

In Sect. 4, we need to know the effect of the $M^{(3)}$, relation (19), as a Lagrangian term. By using the relations

$$
\begin{gathered}
\square R^{2}=2\left(K_{9}+K_{15}\right), \\
\square\left(R_{\mu \nu} R^{\mu \nu}\right)=2\left(K_{10}+K_{16}\right), \\
\square\left(R_{\mu \nu \rho \tau} R^{\mu \nu \rho \tau}\right)=2\left(K_{11}+K_{17}\right), \\
\left(R^{\mu \nu} R_{; \mu}\right)_{; \nu}=K_{12}+\frac{1}{2} K_{15}, \\
\left(R_{\mu \nu} R^{\mu \rho}\right)_{; \rho}^{\nu}=K_{4}-K_{5}+K_{12}+K_{13}+\frac{1}{4} K_{15}
\end{gathered}
$$

and

$$
\left(R_{\mu \nu ; \rho} R^{\rho \mu \nu \lambda}\right)_{; \lambda}=\frac{1}{2} K_{6}-\frac{1}{2} K_{7}-K_{8}-\frac{1}{4} K_{11}+K_{13}-K_{16}
$$

relation (19) reads as

$$
\begin{aligned}
M^{(3)}= & -\frac{1}{2}\left[\left(12 b_{1}+b_{2}\right) R^{2}+2\left(2 b_{2}+b_{5}\right) R_{\mu \nu} R^{\mu \nu}+\left(4 b_{3}+\frac{1}{2} b_{6}\right) R_{\mu \nu \lambda \tau} R^{\mu \nu \lambda \tau}\right]_{; \rho} \rho \\
& -\left(2 b_{2}+b_{5}\right)\left(R^{\mu \nu} R_{; \mu}\right)_{; \nu}-\left(3 b_{4}-2 b_{5}\right)\left(R_{\mu \nu} R^{\mu \rho}\right)_{; \rho}{ }^{\nu}+2\left(b_{5}+b_{6}\right)\left(R_{\mu \nu ; \rho} R^{\rho \mu \nu \lambda}\right)_{; \lambda} .(A)
\end{aligned}
$$

Obviously all of the terms are complete divergences. Therefore, with careful attention about the appropriate boundary conditions (that need to be applied in the process of the variation when a function is up to the fourth order jet-prolongation of the metric), one can easily show that these terms, and hence the $M^{(3)}$, give no contribution to the variation of the corresponding action. 


\section{- The $K_{9}$ To $K_{17}$ As Lagrangian Terms}

In Sect. 5, the effect of each linearly independent term of the $K_{9}$ up to $K_{17}$, as a third order Lagrangian term, is needed. For this purpose, using the null effect of complete divergences (A.13)(A.18) in the variation of their corresponding action and that, the term $K_{14}$ is itself a complete divergence, we obtain 33 the following identities, i.e.

$$
\begin{gathered}
G_{\alpha \beta}^{\left(K_{11}\right)} \equiv-4 G_{\alpha \beta}^{\left(K_{4}\right)}+4 G_{\alpha \beta}^{\left(K_{5}\right)}+2 G_{\alpha \beta}^{\left(K_{6}\right)}-2 G_{\alpha \beta}^{\left(K_{7}\right)}-4 G_{\alpha \beta}^{\left(K_{8}\right)}-G_{\alpha \beta}^{\left(K_{9}\right)}+4 G_{\alpha \beta}^{\left(K_{10}\right)}, \\
G_{\alpha \beta}^{\left(K_{12}\right)} \equiv \frac{1}{2} G_{\alpha \beta}^{\left(K_{9}\right)}, \\
G_{\alpha \beta}^{\left(K_{13}\right)} \equiv-G_{\alpha \beta}^{\left(K_{4}\right)}+G_{\alpha \beta}^{\left(K_{5}\right)}-\frac{1}{4} G_{\alpha \beta}^{\left(K_{9}\right)}, \\
G_{\alpha \beta}^{\left(K_{14}\right)} \equiv 0, \\
G_{\alpha \beta}^{\left(K_{15}\right)} \equiv-G_{\alpha \beta}^{\left(K_{9}\right)}, \\
G_{\alpha \beta}^{\left(K_{16}\right)} \equiv-G_{\alpha \beta}^{\left(K_{10}\right)}
\end{gathered}
$$

and

$$
G_{\alpha \beta}^{\left(K_{17}\right)} \equiv-G_{\alpha \beta}^{\left(K_{11}\right)}
$$

Relation (A.20) has already been used in Ref. [47] (the last relation on its page number 612, after adapting the sign convention and substituting the necessary relations) and Ref. [19] (the first relation of their relation (14)). However, consideration caution has to be exercised in applying identities in general, and identity (A.20) in particular, for there are associated with them some difficulties, but nonetheless they throw some important effects on the effective actions.

Thus, it appears that among the $K_{9}$ to $K_{17}$ Lagrangian terms, it suffices to derive only the metric variation of the $K_{9}$ and $K_{10}$ terms; indeed, we mean that

$$
\delta \int \sum_{i=1}^{17} b_{i} K_{i} \sqrt{-g} d^{D} x=\delta \int \sum_{i=1}^{10} b_{i}^{\prime} K_{i} \sqrt{-g} d^{D} x,
$$

where

$$
\begin{array}{lll}
b_{1}^{\prime}=b_{1}, & b_{2}^{\prime}=b_{2}, & b_{3}^{\prime}=b_{3}, \\
b_{4}^{\prime}=b_{4}-4 b_{11}-b_{13}+4 b_{17}, & b_{5}^{\prime}=b_{5}+4 b_{11}+b_{13}-4 b_{17}, & b_{6}^{\prime}=b_{6}+2 b_{11}-2 b_{17}, \\
b_{7}^{\prime}=b_{7}-2 b_{11}+2 b_{17}, & b_{8}^{\prime}=b_{8}-4 b_{11}+4 b_{17}, & b_{9}^{\prime}=b_{9}-b_{11}+b_{12} / 2-b_{13} / 4-b_{15}+b_{17}, \\
b_{10}^{\prime}=b_{10}+4 b_{11}-b_{16}-4 b_{17} . & & \text { (A.28) }
\end{array}
$$

Hence, as it is customary to write a Lagrangian in terms of its effective one, one can write

$$
\sum_{i=1}^{17} b_{i} K_{i} \stackrel{\text { eff }}{=} \sum_{i=1}^{10} b_{i}^{\prime} K_{i}
$$

where also the word "effective" is often not mentioned. However, as explained in the text, for being able to apply the trace analogy for an effective total third order Lagrangian, we, in practice, need to consider the $K_{1}$ to $K_{11}$ terms, and actually neglect identity (A.20).

The $K_{9}, K_{10}$ and $K_{11}$ Lagrangian terms give

$$
G_{\alpha \beta}^{\left(K_{9}\right)}=\left[2(\square R) R_{\alpha \beta}-2(\square R)_{; \alpha \beta}-R_{; \alpha} R_{; \beta}\right]-\frac{1}{2} g_{\alpha \beta}\left(-4 K_{14}-K_{15}\right),
$$

\footnotetext{
${ }^{33}$ Here, one actually means that the identity holds between the functional derivatives, i.e., for example, $\delta\left(K_{16} \sqrt{-g}\right) / \delta g^{\alpha \beta} \equiv-\delta\left(K_{10} \sqrt{-g}\right) / \delta g^{\alpha \beta}$.
} 


$$
G_{\alpha \beta}^{\left(K_{10}\right)}=R_{\alpha \beta}^{\left(K_{10}\right)}-\frac{1}{2} g_{\alpha \beta}\left(-2 K_{6}+2 K_{7}+4 K_{8}+2 K_{10}+K_{11}-4 K_{13}-K_{14}+5 K_{16}\right)
$$

and

$$
G_{\alpha \beta}^{\left(K_{11}\right)}=R_{\alpha \beta}^{\left(K_{11}\right)}-\frac{1}{2} g_{\alpha \beta}\left(-K_{17}\right)
$$

where 34

$$
\text { Trace } R_{\alpha \beta}^{\left(K_{9}\right)}=\frac{1}{3}\left(2 K_{9}-2 K_{14}-K_{15}\right) \text {, }
$$

$\operatorname{Trace} R_{\alpha \beta}^{\left(K_{10}\right)}=\frac{1}{3}\left(2 K_{4}-2 K_{5}-2 K_{6}+2 K_{7}+4 K_{8}+4 K_{10}+K_{11}-2 K_{13}-\frac{1}{2} K_{15}+5 K_{16}\right)$ and

$$
\text { Trace } R_{\alpha \beta}^{\left(K_{11}\right)}=\frac{1}{3}\left(8 K_{6}-8 K_{7}-16 K_{8}-4 K_{10}+16 K_{13}+2 K_{14}-20 K_{16}+K_{17}\right) .
$$

\section{Appendix B: Dimensional Dependent Constraints For $L_{\text {total }}^{(3)}$}

In this appendix, we perform the analogy for the appearance of the $G_{(\text {total }) \alpha \beta}^{(3 b)}$ in accord with part (b), where

$$
G_{(\text {total }) \alpha \beta}^{(3 b)}=G_{(\text {generic }) \alpha \beta}^{(3 b)}+b_{9} G_{\alpha \beta}^{\left(K_{9}\right)}+b_{10} G_{\alpha \beta}^{\left(K_{10}\right)}+b_{11} G_{\alpha \beta}^{\left(K_{11}\right)}
$$

and similar expressions for $R_{(\text {total }) \alpha \beta}^{(3 b)}$ and $R_{\text {total }}^{(3 b)}$. The $G_{(\text {generic }) \alpha \beta}^{(3 b)}$ is given by relation (18) and the Euler-Lagrange expressions of the $K_{9}$ to $K_{11}$ Lagrangian terms (that are given by relations (A.30)(A.32) ) must be rewritten in accord with part (b). By imposing the condition Trace $R_{(\text {total }) \alpha \beta}^{(3 b)}=$ $R_{\text {total }}^{(3 b)}$, and performing the bulk calculations, we find that the trace relation, for non-zero coefficients, holds if and only if

$$
\begin{aligned}
b_{5}= & {\left[-12(D-1)^{2} b_{1}-\left(3 D^{2}+3 D-4\right) b_{2} / 3-4(3 D-5) b_{3} / 3-b_{4}\right] /(D-1), } \\
b_{6}= & {\left[6(D-1)^{2} b_{1}-\left(3 D^{2}-19 D+14\right) b_{2} / 6-2(D+1) b_{3} / 3-\left(3 D^{2}-7 D+2\right) b_{4} / 4\right] /(D-1), } \\
b_{7}= & (D-2)\left[3(D-1)^{2} b_{1}+\left(5 D^{2}+3 D-6\right) b_{2} / 12+\left(3 D^{2}-7 D+6\right) b_{3} / 3\right. \\
& \left.\quad+\left(D^{2}+3 D-2\right) b_{4} / 8\right] /[2(D-1)], \\
& \quad \\
b_{8}= & 4(D-1)^{2} b_{1}+(D-1)(D+2) b_{2} / 3+4(D-1) b_{3} / 3+(D-2) b_{4} / 2, \\
b_{9}= & \left(b_{2} / 6-2 b_{3} / 3+b_{4} / 4\right) /(D-1), \\
b_{10}= & -2(D-1) b_{9}, \\
b_{11}= & (D-1)(D-2) b_{9} / 2=-(D-2) b_{10} / 4 .
\end{aligned}
$$

If one sets one of the $b_{9}$ or $b_{10}$ or $b_{11}$ zero, then constraint (B.2) will reduce to constraint (40). As mentioned in the text, the authors of Ref. [19] have also derived almost the same relations through a proportionality parameter, say $u$. Indeed, if we set the $b_{13}=0$ and substitute for their proportional parameter in terms of the other coefficients, namely $u=\left[6(D-1)^{2} b_{1}-\left(D^{2}-8 D+6\right) b_{2} / 3+4 D(D-\right.$ $\left.2) b_{3} / 3-D(D-2) b_{4} / 2\right] /(D-1)$, in their relation (B15), it will reduce to constraint (B.2).

\footnotetext{
${ }^{34}$ As we will not need the exact expressions of the $R_{\alpha \beta}^{\left(K_{10}\right)}$ and $R_{\alpha \beta}^{\left(K_{11}\right)}$, to reduce the amount of calculations we have derived their traces almost from the beginning. Implicitly, the appearances of the Euler-Lagrange expressions (A.4) A.8) and (A.30) have been written according to part (a).
} 


\section{References}

[1] Farhoudi, M. "Lovelock tensor as generalized Einstein tensor", Gen. Rel. Grav. 41 (2009), 117129.

[2] Lovelock, D. "The Einstein tensor and its generalizations", J. Math. Phys. 12 (1971), 498-501; "The four dimensionality of space and the Einstein tensor", ibid. 13 (1972), 874-876;

Lovelock, D. \& Rund, H., Tensors, Differential Forms and Variational Principles, (John Wiley \& Sons, New York, 1975);

Briggs, C.C. "Some possible features of general expressions for Lovelock tensors and for the coefficients of Lovelock Lagrangians up to the $15^{\text {th }}$ order in curvature (and beyond)", gr-qc/9808050.

[3] Farhoudi, M. "Classical trace anomaly", Int. J. Mod. Phys. D 14 (2005), 1233-1250.

[4] Duff, M.J. "Twenty years of the Weyl anomaly", Class. Quant. Grav. 11 (1994), 1387-1403.

[5] Deser, S. "Conformal Anomalies - Recent Progress", Helv. Phys. Acta 69 (1996), 570-581.

[6] Asorey, M., Gorbar, E.V. \& Shapiro, I.L. "Universality and ambiguities of the conformal anomaly", Class. Quant. Grav. 21 (2003), 163-178.

[7] Duff, M.J. "Supergravity, Kaluza-Klein and superstrings" in Proc. 11 $1^{\text {th }}$ General Relativity and Gravitation, Stockholm, 1986, Ed. M.A.H. MacCallum (Cambridge University Press, Cambridge, 1987), pp. 18-60.

[8] Birrell, N.D. \& Davies, P.C.W., Quantum Fields in Curved Space, (Cambridge University Press, Cambridge, 1982).

[9] Vilkovisky, G.A. "Effective action in quantum gravity", Class. Quant. Grav. 9 (1992), 895-904.

[10] Farhoudi, M. "On higher order gravities, their analogy to GR, and dimensional dependent version of Duff's trace anomaly relation", Gen. Rel. Grav. 38 (2006), 1261-1284.

[11] Capozziello, S. \& De Laurentis, M. "Extended theories of gravity", Phys. Rep. 509 (2011), $167-321$.

[12] Duff, M.J. "Observations on conformal anomalies", Nucl. Phys. B125 (1977), 334-348.

[13] Deser, S. \& Schwimmer, A. "Geometric classification of conformal anomalies in arbitrary dimensions", Phys. Lett. B309 (1993), 279-284.

[14] Boulanger, N. "Algebraic classification of Weyl anomalies in arbitrary dimensions", Phys. Rev. Lett. 98 (2007), 261302.

[15] Fulton, T., Rohrlich, F. \& Witten, L. "Conformal invariance in physics", Rev. Mod. Phys. 34 (1962), 442-457.

[16] Quiros, I. "The Weyl anomaly and the nature of the background geometry", $g r-q c / 0011056$.

[17] Bonora, L., Pasti, P. \& Bregola, M. "Weyl cocycles", Class. Quant. Grav. 3 (1986), 635-649.

[18] Bonora, L., Cotta-Ramusino, P. \& Reina, C. "Conformal anomaly and cohomology", Phys. Lett. B126 (1983), 305-308;

Nojiri, S., Odintsov, S.D. \& Ogushi, S. "Holographic renormalization group and conformal anomaly for $\mathrm{AdS}_{9} / \mathrm{CFT}_{8}$ correspondence", Phys. Lett. B500 (2001), 199-208.

[19] Oliva, J. \& Ray, S. "Classification of six derivative Lagrangians of gravity and static spherically symmetric solutions", Phys. Rev. D82 (2010), 124030. 
[20] Wald, R.M., General Relativity, (The University of Chicago Press, Chicago, 1984).

[21] Zwiebach, B. "Curvature squared terms and string theories", Phys. Lett. B156 (1985), 315-317; Zumino, B. "Gravity theories in more than four dimensions", Phys. Rep. 137 (1986), 109-114.

[22] Isham, C.J. "Quantum gravity" in Proc. 11 ${ }^{\text {th }}$ General Relativity and Gravitation, Stockholm, 1986, Ed. M.A.H. MacCallum (Cambridge University Press, Cambridge, 1987), pp. 99-129; "Structural issues in quantum gravity", gr-qc/9510063.

[23] Nojiri, S. \& Odintsov, S.D. "Introduction to modified gravity and gravitational alternative for dark energy", Int. J. Geom. Methods Mod. Phys. 4 (2007), 115-146;

Schmidt, H.-J. "Fourth order gravity: equations, history, and applications to cosmology", Int. J. Geom. Methods Mod. Phys. 4 (2007), 209-248;

Capozziello, S., De Laurentis, M. \& Faraoni, V. "A bird's eye view of $f(R)$-gravity", grqc/0909.4672;

Sotiriou, T.P. \& Faraoni, V. " $f(R)$ theories of gravity", Rev. Mod. Phys. 82 (2010), 451-497;

Capozziello, S. \& Faraoni, V., Beyond Einstein Gravity: A Survey of Gravitational Theories for Cosmology and Astrophysics, (Springer, London, 2011);

Nojiri, S. \& Odintsov, S.D. "Unified cosmic history in modified gravity: From $F(R)$ theory to Lorentz non-invariant models", Phys. Rep. 505 (2011), 59-144;

Clifton, T., Ferreira, P.G., Padilla, A. \& Skordis, C. "Modified gravity and cosmology", Phys. Rep. 513 (2012), 1-189.

[24] Müller-Hoissen, F. "Spontaneous compactification with quadratic and cubic curvature term", Phys. Lett. B163 (1985), 106-110.

[25] Wheeler, J.T. "Symmetric solutions to the Gauss-Bonnet extended Einstein equations", Nucl. Phys. B268 (1986), 737-746.

[26] Farhoudi, M., Non-linear Lagrangian Theories of Gravitation, (Ph.D. Thesis, Queen Mary \& Westfield College, University of London, 1995).

[27] Fulling, S.A., King, R.C., Wybourne, B.G. \& Cummins, C.J. "Normal forms for tensor polynomials: I. The Riemann tensor", Class. Quant. Grav. 9 (1992), 1151-1197.

[28] Matyjasek, J., Telecka, M. \& Tryniecki, D. "Higher dimensional black holes with a generalized gravitational action", Phys. Rev. D73 (2006), 124016;

Oliva, J. \& Ray, S. "A new cubic theory of gravity in five dimensions: black hole, Birkhoff's theorem and C-function", Class. Quant. Grav. 27 (2010), 225002;

Myers, R.C. \& Robinson, B. "Black holes in quasi-topological gravity", gr-qc/1003.5357.

[29] Gottlöber, S., Schmidt, H.--J. \& Starobinsky, A.A. "Sixth-order gravity and conformal transformations", Class. Quant. Grav. 7 (1990), 893-900.

[30] Berkin, A.L. \& Maeda, K. "Effects of $R^{3}$ and $R \square R$ terms on $R^{2}$ inflation", Phys. Lett. B245 (1990), 348-354.

[31] Décanini, Y. \& Folacci, A. "Irreducible forms for the metric variations of the action terms of sixth-order gravity and approximated stress-energy tensor", Class. Quant. Grav. 24 (2007), 4777-4799.

[32] Metsaev, R.R. \& Tseytlin, A.A. "Curvature cubed terms in string theory effective actions", Phys. Lett. B185 (1987), 52-58;

Ketov, S.V. "The string generated correction to Einstein gravity from the sigma model approach", Gen. Rel. Grav. 22 (1990), 193-202. 
[33] Erdmenger, J. "Conformally covariant differential operators: properties and applications", Class. Quant. Grav. 14 (1997), 2061-2084.

[34] Barvinsky, A.O., Gusev, Yu.V., Zhytnikov, V.V. \& Vilkovisky, G.A. "Covariant perturbation theory (IV). Third order in the curvature", hep-th/0911.1168.

[35] Chern, S.-S. "A simple intrinsic proof of the Gauss-Bonnet formula for closed Riemannian manifolds", Ann. Math. 45 (1944), 747-752; "On the curvatura integra in a Riemannian manifold", ibid. 46 (1945), 674-684;

Kobayashi, S. \& Nomizu, K., Foundations of Differential Geometry, Vol. II, (Wiley Interscience, New York, 1969);

Spivak, M., A Comprehensive Introduction to Differential Geometry, Vol. 5, (Publish or Perlish Inc., Delaware, $2^{\text {nd }}$ ed. 1979).

[36] Fefferman, C. \& Graham, C.R. "Conformal invariants", in The Mathematical Heritage of Élie Cartan, Lyon 1984, Astérisque, hors série (1985), pp. 95-116.

[37] Karakhanyan, D.R., Manvelyan, R.P. \& Mkrtchyan, R.L. "Trace anomalies and cocycles of Weyl and diffeomorphisms groups", Mod. Phys. Lett. A11 (1996), 409-422.

[38] Parker, T. \& Rosenberg, S. "Invariants of conformal Laplacians", J. Diff. Geom. 25 (1987), 199-222.

[39] Arakelyan, T., Karakhanyan, D.R., Manvelyan, R.P. \& Mkrtchyan, R.L. "Trace anomalies and cocycles of Weyl group", preprint 1995, Yerevan Physics Institute, Armenia.

[40] Odintsov, S.D. \& Romeo A. "Conformal sector in $D=6$ quantum gravity", Mod. Phys. Lett. A9 (1994), 3373-3381.

[41] Capper, D.M. \& Duff, M.J. "Trace anomalies in dimensional regularization", Nuovo Cim. 23A (1974), 173-183.

[42] Nojiri, S. \& Odintsov, S.D. "Quantum dilatonic gravity in $d=2,4$ and 5 dimensions", Int. J. Mod. Phys. A16 (2001), 1015-1108.

[43] Deser, S., Duff, M.J. \& Isham, C.J. "Non-local conformal anomalies", Nucl. Phys. B111 (1976), 45-55;

Christensen, S.M. "Regularization, renormalization, and covariant geodesic point separation", Phys. Rev. D17 (1978), 946-963.

[44] Gibbons, G.W. "Quantum field theory in curved space-time" in General Relativity: An Einstein Centenary Survey, Ed. S.W. Hawking \& W. Israel (Cambridge University Press, Cambridge, 1979), pp. 1-23.

[45] Avramidi, I.G. "Covariant techniques for computation of the heat kernel", Rev. Math. Phys. 11 (1999), 947-980.

[46] Amsterdamski, P., Berkin, A.L. \& O'Connor, D.J. "b $\mathbf{8}$ 'Hamidew' coefficient for a scalar field", Class. Quant. Grav. 6 (1989), 1981-1991.

[47] Gilkey, P.B. "The spectral geometry of a Riemannian manifold", J. Diff. Geom. 10 (1975), 601-618.

[48] DeWitt, B.S. "Dynamical theory of groups and fields" in Relativity, Groups and Topology, Les Houches Summer School in 1963, Ed. C. DeWitt \& B. DeWitt (Gordon and Breach, New York, 1964), pp. 587-820. It is also published as a book with the same title by Blackie \& Son Limited, 1965. 
[49] Avramidi, I.G. \& Schimming, R. "Algorithms for the calculation of the heat kernel coefficients" in Quantum Field Theory Under The Influence of External Conditions, Ed. M. Bordag, TeubnerTexte zur Physik, Vol. 30, (Teubner, Stuttgart, 1996), pp. 150-162, hep-th/9510206].

[50] Schimming, R. "Calculation of the heat kernel coefficients" in Analysis, Geometry and Groups: A Riemann Legacy Volume, Ed. H.M. Srivastava \& T.M. Rassias (Hadronic Press, Palm Harbor, Florida, 1993), pp. 627-656.

[51] Gilkey, P.B. "Recursion relations and the asymptotic behavior of the eigenvalues of the Laplacian", Compositio Math. 38 (1979), 201-240.

[52] Parker, L. \& Toms, D.J. "New form for the coincidence limit of the Feynman propagator, or heat kernel, in curved space-time", Phys. Rev. D31 (1985), 953-956.

[53] Buchdahl, H.A. "Über die Variationsableitung von fundamental-invarianten beliebig hoher Ordnung" (On the variational derivative of fundamental invariants of arbitrarily high order), Acta Math. 85 (1951), 63-72. 